\title{
Search for top and bottom squarks from gluino pair production in final states with missing transverse energy and at least three $b$-jets with the ATLAS detector
}

\author{
The ATLAS Collaboration ${ }^{\star}$ \\ CERN, 1211 Geneva 23, Switzerland
}

Received: 19 July 2012 / Revised: 10 September 2012 / Published online: 6 October 2012

(C) CERN for the benefit of the ATLAS collaboration 2012. This article is published with open access at Springerlink.com

\begin{abstract}
This letter reports the results of a search for top and bottom squarks from gluino pair production in $4.7 \mathrm{fb}^{-1}$ of $p p$ collisions at $\sqrt{s}=7 \mathrm{TeV}$ using the ATLAS detector at the LHC. The search is performed in events with large missing transverse momentum and at least three jets identified as originating from a $b$-quark. Exclusion limits are presented for a variety of gluino-mediated models with gluino masses up to $1 \mathrm{TeV}$ excluded.
\end{abstract}

Supersymmetry (SUSY) [1-9] provides an extension of the Standard Model (SM) which resolves the hierarchy problem [10-13] by introducing supersymmetric partners of the known bosons and fermions. In the framework of the $R$ parity conserving minimal supersymmetric extension of the SM (MSSM) [14-18], SUSY particles are produced in pairs and the lightest supersymmetric particle (LSP) is stable, providing a possible candidate for dark matter. In a large variety of models, the LSP is the lightest neutralino $\left(\tilde{\chi}_{1}^{0}\right)$. The colored superpartners of quarks and gluons, the squarks $(\tilde{q})$ and gluinos $(\tilde{g})$, if not too heavy, would be produced in strong interaction processes at the Large Hadron Collider (LHC) and decay via cascades ending with the LSP. The undetected LSP results in missing transverse momentumwhose magnitude is referred to as $E_{\mathrm{T}}^{\text {miss }}$ - while the rest of the cascade yields final states with multiple jets and possibly leptons. In the MSSM, the right-handed and left-handed squarks, $\tilde{q}_{R}$ and $\tilde{q}_{L}$, can mix to form two mass eigenstates $\tilde{q}_{1}$ and $\tilde{q}_{2}$. The mixing effect is proportional to the masses of the SM fermion partners and can therefore be large for the third generation. This may lead to the lightest sbottom $\left(\tilde{b}_{1}\right)$ and stop $\left(\tilde{t}_{1}\right)$ mass eigenstates being much lighter than the other squarks. As a consequence, $\tilde{b}_{1}$ and $\tilde{t}_{1}$ could be produced with relatively large cross sections at the LHC, ei-

^e-mail: atlas.publications@cern.ch ther directly in pairs, or through $\tilde{g} \tilde{g}$ production followed by $\tilde{g} \rightarrow \tilde{b}_{1} b$ or $\tilde{g} \rightarrow \tilde{t}_{1} t$ decays.

This letter extends the search for gluino-mediated $\tilde{b}_{1}$ and $\tilde{t}_{1}$ production at ATLAS reported earlier [19], which used $2.05 \mathrm{fb}^{-1}$ of data collected in the first half of 2011 at a center-of-mass energy of $7 \mathrm{TeV}$. The present analysis comprises the full 2011 dataset of $4.7 \mathrm{fb}^{-1}$ and adopts an improved selection that requires large $E_{\mathrm{T}}^{\text {miss }}$, no electron or muon and at least three jets identified as originating from $b$-quarks ( $b$-jets) in the final state. Results are interpreted in four simplified models where sbottoms or stops are the only squarks produced in the gluino decays, leading to final states with four $b$-quarks. Searches in similar scenarios have also been reported by the CMS Collaboration [20].

The ATLAS detector [21] consists of inner tracking devices surrounded by a superconducting solenoid, electromagnetic and hadronic calorimeters and a muon spectrometer with a toroidal magnetic field. The inner detector provides precision tracking of charged particles for $|\eta|<2$. . $^{1}$ It is immersed in a $2 \mathrm{~T}$ magnetic field from the solenoid and consists of a silicon pixel detector, a silicon strip detector and a straw tube tracker that also provides transition radiation measurements for electron identification. The calorimeter system covers the pseudorapidity range $|\eta|<4$.9. It is composed of sampling calorimeters with either liquid argon (LAr) or scintillating tiles as the active medium. The muon spectrometer has separate trigger and high-precision tracking chambers which provide muon identification and momentum measurement for $|\eta|<2.7$.

\footnotetext{
${ }^{1}$ ATLAS uses a right-handed coordinate system with its origin at the nominal interaction point (IP) in the center of the detector and the $z$ axis along the beam pipe. The $x$-axis points from the IP to the center of the LHC ring, and the $y$ axis points upward. Cylindrical coordinates $(r, \phi)$ are used in the transverse plane, $\phi$ being the azimuthal angle around the beam pipe. The pseudorapidity is defined in terms of the polar angle $\theta$ as $\eta=-\ln \tan (\theta / 2)$. The distance $\Delta R$ in the $\eta-\phi$ space is defined as $\Delta R=\sqrt{(\Delta \eta)^{2}+(\Delta \phi)^{2}}$.
} 
Samples of simulated events are used for the description of the background and to model the SUSY signal. The dominant sources of background come from events with $b$-quarks in the final state. Monte Carlo (MC) samples of $t \bar{t}, W / Z$ and diboson events produced in association with light- and heavy-flavor jets are generated with ALPGEN [22] and the parton distribution function (PDF) set CTEQ6L1 [23]. These samples are generated with different maximum numbers of additional partons at the matrixelement level. Diboson samples are generated with up to three additional partons, $t \bar{t}+$ jet and $Z\left(\rightarrow \ell^{+} \ell^{-}\right)+$jets $(\ell=e, \mu, \tau)$ samples with up to five additional partons, and $W(\rightarrow \ell v)+$ jets and $Z(\rightarrow \bar{v} v)+$ jet samples with up to six additional partons. Single top quark production is simulated with MC@NLO [24] using the next-to-leading-order (NLO) PDF set CTEQ6 . 6 [25]. The fragmentation and hadronization for the ALPGEN and MC@NLO samples are performed with HERWIG [26, 27], using JIMMY [28] for the underlying event. Samples of $t \bar{t}+W, t \bar{t}+Z$ and $t \bar{t}+W W$ events are generated with MADGRAPH [29] interfaced to PYTHIA [30]. The signal samples are generated using Herwig++ [31]. The MC samples are processed through the ATLAS detector simulation [32] based on GEANT4 [33] taking into account the effect of multiple $p p$ interactions per bunch crossing. For the comparison with data, all SM background cross sections are normalized to the results of higher-order calculations when available, using the same values as in Ref. [19].

Jets are reconstructed from three-dimensional calorimeter energy clusters using the anti- $k_{t}$ jet algorithm $[34,35]$ with a radius parameter of 0.4 . The measured jet energy is corrected for inhomogeneities and for the non-compensating nature of the calorimeter by using $p_{\mathrm{T}^{-}}$and $\eta$-dependent correction factors, and additional corrections for pile-up are applied [36]. Jets are required to have $p_{T}>20 \mathrm{GeV}$, and are reconstructed in the range $|\eta|<4$.9. Events are rejected if they include jets failing the quality criteria described in Ref. [36], or if there is any selected jet with $|\eta|<2$ for which the scalar sum of the transverse momenta of its associated tracks is less than $5 \%$ of the jet $p_{\mathrm{T}}$. A neural-network-based algorithm [37] is used to identify jets containing a $b$-hadron decay. This uses as input the output weights of different algorithms exploiting the impact parameter of the inner detector tracks, the secondary vertex reconstruction and the topology of $b$ - and $c$-hadron decays inside the jet. Three operating points are used, corresponding to efficiencies of $60 \%, 70 \%$ and $75 \%$ for tagging $b$-jets in a MC sample of $t \bar{t}$ events. In all cases the tagging rate is less than $2 \%$ for light-quark and gluon jets, $10 \%$ for $\tau$ leptons decaying hadronically and $25 \%$ for $c$-quark jets. The $b$-jets are identified within the nominal acceptance of the inner detector $(|\eta|<2.5)$ and are required to have $p_{\mathrm{T}}>30 \mathrm{GeV}$. To compensate for the differences between the $b$-tagging efficiency and the mistag rates in data and MC simulation, $b$-tagging
Table 1 Definition of the five signal regions based on the number of jets $\left(N_{J}\right)$, the $E_{\mathrm{T}}^{\text {miss }}, m_{\text {eff }}$ requirements and the $b$-tagging operating point (OP)

\begin{tabular}{lllll}
\hline SR & $N_{J}$ & $E_{\mathrm{T}}^{\text {miss }}$ & $m_{\text {eff }}$ & $b$-tag OP \\
\hline SR4-L & $\geq 4 \mathrm{j}$ & $>160 \mathrm{GeV}$ & $>500 \mathrm{GeV}$ & $60 \%$ \\
SR4-M & $\geq 4 \mathrm{j}$ & $>160 \mathrm{GeV}$ & $>700 \mathrm{GeV}$ & $60 \%$ \\
SR4-T & $\geq 4 \mathrm{j}$ & $>160 \mathrm{GeV}$ & $>900 \mathrm{GeV}$ & $70 \%$ \\
SR6-L & $\geq 6 \mathrm{j}$ & $>160 \mathrm{GeV}$ & $>700 \mathrm{GeV}$ & $70 \%$ \\
SR6-T & $\geq 6 \mathrm{j}$ & $>200 \mathrm{GeV}$ & $>900 \mathrm{GeV}$ & $75 \%$ \\
\hline
\end{tabular}

Common criteria: lepton veto, $p_{\mathrm{T}}^{j_{1}}>130 \mathrm{GeV}, \geq 3 b$-jets, $E_{\mathrm{T}}^{\mathrm{miss}} / m_{\mathrm{eff}}>0.2, \Delta \phi_{\min }>0.4$

Table 2 Definition of the four control regions used to estimate the $t \bar{t}+$ jets background

\begin{tabular}{llll}
\hline CR & $N_{J}$ & $b$-tag OP & corresponding SR \\
\hline CR4-60 & $\geq 4 \mathrm{j}$ & $60 \%$ & SR4-L, SR4-M \\
CR4-70 & $\geq 4 \mathrm{j}$ & $70 \%$ & SR4-T \\
CR6-70 & $\geq 6 \mathrm{j}$ & $70 \%$ & SR6-L \\
CR6-75 & $\geq 6 \mathrm{j}$ & $75 \%$ & SR6-T \\
\hline
\end{tabular}

Common criteria: lepton veto, $p_{\mathrm{T}}^{j_{1}}>130 \mathrm{GeV},=2 b$-jets, $E_{\mathrm{T}}^{\mathrm{miss}} / m_{\mathrm{eff}}>0.2, \Delta \phi_{\min }>0.4, E_{\mathrm{T}}^{\mathrm{miss}}>160 \mathrm{GeV}, m_{\mathrm{eff}}>500 \mathrm{GeV}$

scale factors are applied to each jet in the simulations, as described in Refs. [37-39].

Electrons are reconstructed from energy clusters in the electromagnetic calorimeter matched to a track in the inner detector. Electron candidates are required to have $p_{\mathrm{T}}>$ $20 \mathrm{GeV}$ and $|\eta|<2.47$ and must satisfy the "medium" selection criteria described in Ref. [40]. Muons candidates are identified using a match between an extrapolated inner detector track and one or more track segments in the muon spectrometer, and are required to have $p_{\mathrm{T}}>10 \mathrm{GeV}$ and $|\eta|<2.4$.

Since electrons are also reconstructed as jets, jets within a distance of $\Delta R=0.2$ of an electron candidate are rejected. Furthermore, any lepton candidate with a distance $\Delta R<0.4$ to the closest remaining jet is discarded. Events containing any remaining electrons and muons are vetoed in the control and signal regions defined in Tables 1 and 2.

The measurement of the missing transverse momentum two-dimensional vector (and its magnitude $E_{\mathrm{T}}^{\text {miss }}$ ) is based on the transverse momenta of all remaining jets with $|\eta|<$ 4.9, all electron and muon candidates and all calorimeter clusters not associated to such objects.

Events are selected using triggers requiring one high $\mathrm{T}$ jet and $E_{\mathrm{T}}^{\mathrm{miss}}$. Different trigger thresholds were used to cope with the increasing luminosity. These triggers are fully efficient for this analysis, which requires one jet with $p_{\mathrm{T}}>$ $130 \mathrm{GeV}$ and $E_{\mathrm{T}}^{\text {miss }}>160 \mathrm{GeV}$ at the offline reconstruction 
stage. Events must pass basic quality criteria to reject detector noise and non-collision backgrounds. They are also required to have a reconstructed primary vertex associated with five or more tracks with $\mathrm{T}>0.4 \mathrm{GeV}$; when more than one such vertex is found, the vertex with the largest summed ${ }_{\mathrm{T}}^{2}$ of the associated tracks is chosen as the hard interaction vertex. Events are required to have at least three $b$-tagged jets, and two jet-multiplicity regions $\left(N_{J} \geq 4\right.$ and $\left.N_{J} \geq 6\right)$ are considered by selecting jets with $|\eta|<2.8$ and $\mathrm{T}>50 \mathrm{GeV}$.

Two variables are calculated from the reconstructed objects to further select the events: $m_{\mathrm{eff}}$ and $\Delta \phi_{\min }$. The effective mass $m_{\text {eff }}$ is defined as the scalar sum of the $E_{\mathrm{T}}^{\text {miss }}$ and the $p_{\mathrm{T}}$ of all selected jets in a given jet-multiplicity region. The $\Delta \phi_{\min }$ is defined as the minimum azimuthal separation between the selected jets and the missing transverse momentum direction. Placing the requirements $\Delta \phi_{\min }>0.4$ and $E_{\mathrm{T}}^{\text {miss }} / m_{\text {eff }}>0.2$ reduces the amount of multi-jet background, where $E_{\mathrm{T}}^{\mathrm{miss}}$ results from mis-reconstructed jets or from neutrinos emitted close to the direction of the jet axis.

Two sets of signal regions are defined which yield good signal sensitivity for the various models and parameter values studied here. They are characterized by having at least four (SR4) or six (SR6) jet candidates, no electron or muon, and are further classified as loose (L), medium (M) or tight (T) depending on the $E_{\mathrm{T}}^{\text {miss }}$ and $m_{\text {eff }}$ thresholds and on the $b$ tagging operating point. The requirements that characterize each signal region are summarized in Table 1.

The main source of reducible background is the production of $t \bar{t}$ events in association with additional jets followed by the leptonic decay of one $W$ boson, where the lepton is not reconstructed or is misidentified as a jet (mainly through the hadronic decays of a $\tau$ lepton). This background is estimated by normalizing the $\mathrm{MC}$ event yield in the signal region to the extrapolated event yield observed in a $t \bar{t}$ dominated control region. Systematic uncertainties that are correlated between the control and the signal regions largely cancel out in this procedure. Additional sources of reducible background are single top, $t \bar{t}+W / Z$ and $W / Z+$ heavyflavor jets. Their contributions are taken from MC simulation and account for $10 \%$ to $20 \%$ of the total background depending on the signal region. The irreducible background $t \bar{t}+b \bar{b}$ is also estimated from MC simulation and accounts for about $10 \%$ of the total background in all signal regions. The reducible contribution from multi-jet events is estimated with a data-driven method, based on a jet response smearing technique [41], and is found to account for less than $5 \%$ of the total background in all signal regions.

Four control regions where the $t \bar{t}+$ jets background accounts for more than $80 \%$ of the total yield are defined by applying the same jet requirements and lepton veto as in the signal regions, but requiring exactly two $b$-jets instead of three or more. The requirements $m_{\mathrm{eff}}>500 \mathrm{GeV}$
Table 3 Expected numbers of SM events and observed data events in the four $t \bar{t}$ control regions. The contribution from $t \bar{t}+$ jets events is taken directly from MC simulation. The column "others" includes the contributions from single top, $t \bar{t}+b \bar{b}, t \bar{t}+W / Z$ and $W / Z+$ jets processes, also estimated from MC simulation, and the contribution from multi-jet events which is estimated with the jet smearing technique and accounts for less than $5 \%$ of the total background. The column "SM" shows the total expected background and is the sum of the columns " $t \bar{t}+$ jets" and "others". The uncertainties include all detector-related systematic uncertainties

\begin{tabular}{lllll}
\hline CR & $t \bar{t}+$ jets & others & SM & data \\
\hline CR4-60 & $330 \pm 90$ & $65 \pm 25$ & $395 \pm 115$ & 402 \\
CR4-70 & $490 \pm 125$ & $100 \pm 35$ & $590 \pm 160$ & 515 \\
CR6-70 & $38 \pm 11$ & $7 \pm 3$ & $45 \pm 13$ & 46 \\
CR6-75 & $40 \pm 12$ & $10 \pm 4$ & $50 \pm 15$ & 52 \\
\hline
\end{tabular}

and $E_{\mathrm{T}}^{\text {miss }}>160 \mathrm{GeV}$ are applied to all control regions to make them kinematically similar to the signal regions, while reducing the contamination from possible SUSY signal events. The definition of the control regions is summarized in Table 2. The numbers of expected SM events in the four control regions, as predicted by the jet smearing technique for multi-jet events and by MC simulation for other processes, are compared to those observed in data in Table 3. The results agree within experimental errors.

The dominant detector-related systematic effects are due to the jet energy scale (JES) and resolution (JER) uncertainties, and the uncertainty on the $b$-tagging efficiency and mistag rates. The JES uncertainty is derived from a combination of simulations, test beam data and in-situ measurements [36], and includes additional uncertainties due to the jet flavor and nearby jets. Uncertainties on the JER are obtained with an in-situ measurement of the jet response asymmetry in di-jet events. These uncertainties on jets are propagated to the $E_{\mathrm{T}}^{\text {miss }}$ measurement, and additional uncertainties on $E_{\mathrm{T}}^{\text {miss }}$ arising from energy deposits not associated with any reconstructed objects are also included. The $b$-tagging uncertainty is evaluated by varying the $\eta-, \mathrm{T}^{-}$and flavordependent scale factors applied to each jet in the simulation within a range that reflects the systematic uncertainty on the measured tagging efficiency and mistag rates.

The systematic uncertainties in the modeling of the $t \bar{t}+$ jets background are assessed as follows: the uncertainty due to the choice of the $\mathrm{MC}$ generator is estimated by comparing the leading-order ALPGEN generator to the MC@NLO generator; the uncertainty due to the factorization and matching scale ambiguities in ALPGEN are estimated by independently varying their nominal settings by factors of one half and two; the uncertainty due to the finite number of additional partons at the matrix-element level is assessed by comparing inclusive ALPGEN samples generated with up to three and up to five extra partons. Finally the PDF uncertainties are estimated using the MSTW2008NNLO PDF set. 
Table 4 Comparison between the results of the fits and the numbers of observed events in the five signal regions. The $t \bar{t}+$ jets event yield predicted by the MC simulation is quoted in parentheses. The column "others" includes the contributions from single top, $t \bar{t}+b \bar{b}, t \bar{t}+W / Z$, $W / Z+$ jets and multi-jet processes. Multi-jet events contribute less than $5 \%$ of the total background. The column "SM" shows the total expected background and is the sum of the columns " $t \bar{t}+$ jets" and "others". The uncertainties include the statistical and systematic uncertainties

\begin{tabular}{lllll}
\hline SR & $\begin{array}{l}t \bar{t}+\text { jets } \\
(\mathrm{MC})\end{array}$ & others & SM & data \\
\hline SR4-L & $\begin{array}{l}33.3 \pm 7.9 \\
(32.6 \pm 15.4)\end{array}$ & $11.1 \pm 4.9$ & $44.4 \pm 10.0$ & 45 \\
SR4-M & $\begin{array}{l}16.4 \pm 4.1 \\
(16.1 \pm 8.4)\end{array}$ & $6.6 \pm 2.9$ & $23.0 \pm 5.4$ & 14 \\
SR4-T & $\begin{array}{l}9.6 \pm 2.1 \\
(11.4 \pm 5.4)\end{array}$ & $3.7 \pm 1.6$ & $13.3 \pm 2.6$ & 10 \\
SR6-L & $\begin{array}{l}10.3 \pm 3.3 \\
(10.0 \pm 6.2)\end{array}$ & $2.4 \pm 1.4$ & $12.7 \pm 3.6$ & 12 \\
SR6-T & $\begin{array}{l}8.3 \pm 2.4 \\
(7.9 \pm 5.3)\end{array}$ & $1.6 \pm 1.1$ & $9.9 \pm 2.6$ & 8 \\
& & & \\
\hline
\end{tabular}

Uncertainties of $100 \%$ are assumed for the multi-jet prediction and for the cross section of $t \bar{t}$ and $W / Z$ production in association with a $b \bar{b}$ pair. For $t \bar{t}+W / Z$ production, an uncertainty of approximatively $70 \%$ has been derived from the variations of the factorization and renormalization scales and from the PDF uncertainties [42].

The $t \bar{t}+$ jets yield in each signal region is extrapolated from the measured number of events in the corresponding control region (as per Table 2) using a fit based on the profile likelihood method [43]. Each pair of control and signal regions is fitted separately, assuming no signal events. The free parameter in each fit is the $t \bar{t}+$ jets overall normalization scale, while the contributions from subdominant background processes are fixed at the expected value. Systematic uncertainties are treated as nuisance parameters constrained with a Gaussian function and correlations are taken into account where appropriate. The results of the fits and the numbers of observed events for each signal region are summarized in Table 4. The fitted values of the normalization factors for $t \bar{t}+$ jets are compatible with one and the main impact of the data-driven estimate is a reduction in the uncertainty by approximately a factor of two. Figure 1 shows the measured $m_{\text {eff }}$ distributions and the MC predictions for the SM backgrounds in each signal region. Also shown are the prediction of two benchmark signal models described below.

The reliability of the MC extrapolation of the $t \bar{t}$ background to larger $b$-jet multiplicities has been checked in validation regions defined with kinematic cuts similar to those used in the control and signal regions, except that exactly one isolated electron or muon is required. The transverse mass of the lepton and the $E_{\mathrm{T}}^{\text {miss }}$ is required to be less than
Table 5 Observed (expected) $95 \%$ CL upper limits on the non-SM contributions to all signal regions. Limits are given on numbers of events and in terms of visible cross sections defined by cross section times kinematic acceptance times experimental efficiency. Systematic uncertainties on the SM background estimation are included in the limits

\begin{tabular}{lll}
\hline SR & \multicolumn{2}{l}{ Obs (exp) 95\% CL upper limit } \\
\cline { 2 - 3 }$N$ events & $\sigma_{\text {vis }}(\mathrm{fb})$ \\
\hline SR4-L & $23.8(23.4)$ & $5.1(5.0)$ \\
SR4-M & $8.6(12.8)$ & $1.8(2.7)$ \\
SR4-T & $7.1(9.2)$ & $1.5(2.0)$ \\
SR6-L & $9.6(10.1)$ & $2.0(2.1)$ \\
SR6-T & $7.1(8.3)$ & $1.5(1.8)$ \\
\hline
\end{tabular}

$100 \mathrm{GeV}$ in all validation regions to minimize the possible contamination from stop production. The extrapolated event yield in the validation regions with at least three $b$-jets from the validation regions with exactly two $b$-jets is found to be consistent with the number of observed events for all $b$ tagging operating points.

The background predictions have been further validated using a data-driven method that simultaneously estimates all SM background contributions with at least one misidentified $b$-jet. This method consists of predicting the number of jets originating from $b$-quarks in each event by solving a system of equations based on the number of $b$-tagged and non $b$ tagged jets in the event, along with the $b$-tagging efficiency and mistag rates. Consistent background predictions with respect to the fit results have been found in all signal regions.

Limits for non-SM signal at $95 \%$ confidence level (CL) are derived by testing the signal plus background hypothesis in each signal region with the $C L_{s}$ prescription [43]. These limits are obtained with fits similar to those used to estimate the background in each signal region, except that the number of observed events in the signal region is added as an input to the fit and a second free parameter for the non-SM signal strength, constrained to be non-negative, is adjusted in the likelihood maximization. This additional free parameter ensures a proper treatment of the expected signal contamination in the control regions when the results are interpreted in the framework of specific SUSY scenarios. Modelindependent upper limits at $95 \% \mathrm{CL}$ on the number of signal events and on the visible cross section (defined as the cross section times kinematic acceptance times experimental efficiency) for non-SM contributions derived for each signal region are given in Table 5 .

These data have been used to derive limits in the parameter space of the following SUSY models.

Gluino-sbottom model MSSM scenarios where the $\tilde{b}_{1}$ is the lightest squark, all other squarks are heavier than the gluino, and $m_{\tilde{g}}>m_{\tilde{b}_{1}}>m_{\tilde{\chi}_{1}^{0}}$, so the branching ratio for 

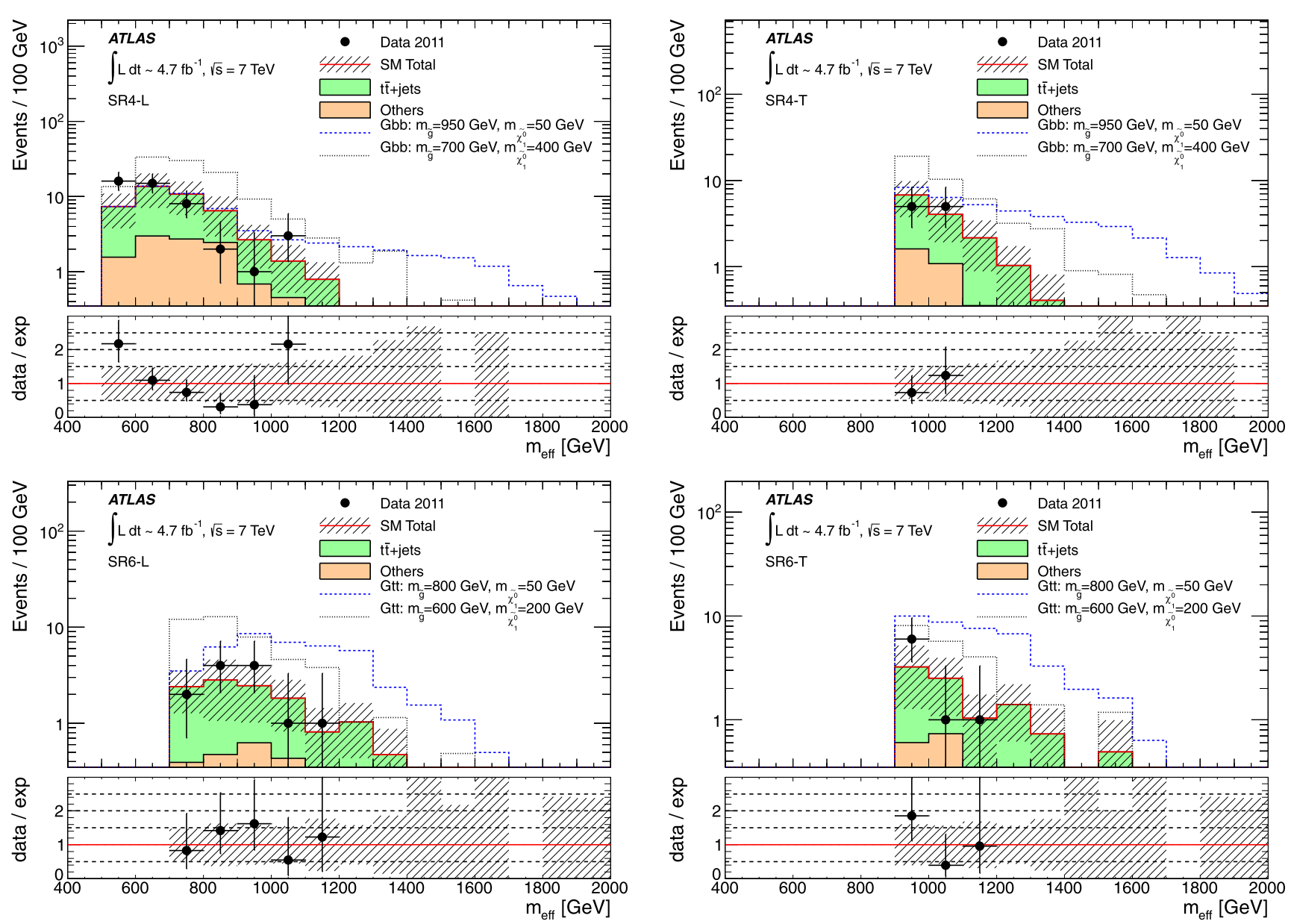

Fig. 1 Distribution of $m_{\text {eff }}$ for SR4-L and SR4-T (top) and SR6-L and SR6-T (bottom). The hatched band shows the systematic uncertainty on the MC prediction, which includes both experimental uncertainties (among which JES and $b$-tagging uncertainties are dominant) and theoretical uncertainties on the background normalization and shape. The label "others" includes the contributions from single top, $t \bar{t}+b \bar{b}$,

$\tilde{g} \rightarrow \tilde{b}_{1} b$ decays is $100 \%$. Sbottoms are produced via $\tilde{g} \tilde{g}$ or by $\tilde{b}_{1} \tilde{b}_{1}$ direct pair production and are assumed to decay exclusively via $\tilde{b}_{1} \rightarrow b \tilde{\chi}_{1}^{0}$, where $m_{\tilde{\chi}_{1}^{0}}$ is set to $60 \mathrm{GeV}$. Exclusion limits are presented in the $\left(m_{\tilde{g}}, m_{\tilde{b}_{1}}\right)$ plane.

Gbb model Simplified scenarios, where $\tilde{b}_{1}$ is the lightest squark but $m_{\tilde{g}}<m_{\tilde{b}_{1}}$. Pair production of gluinos is the only process taken into account since the masses of all other sparticles apart from the $\tilde{\chi}_{1}^{0}$ are set above the $\mathrm{TeV}$ scale. A three-body decay via an off-shell sbottom is assumed for the gluino, yielding a $100 \% \mathrm{BR}$ for the decay $\tilde{g} \rightarrow b \bar{b} \tilde{\chi}_{1}^{0}$. The sbottom mass has no impact on the kinematics of the decay and the exclusion limits are presented in the $\left(m_{\tilde{g}}, m_{\tilde{\chi}_{1}^{0}}\right)$ plane.

Gluino-stop model MSSM scenarios where the $\tilde{t}_{1}$ is the lightest squark, all other squarks are heavier than the gluino,

$t \bar{t}+W / Z, W / Z+$ jets and multi-jet processes. The lower plot in each figure shows the ratio of the observed distribution to that expected for the SM background. Two signal points (with small and large mass splitting between the gluino and the LSP) for the $G b b$ and Gtt models described in the text are overlaid

and $m_{\tilde{g}}>m_{\tilde{t}_{1}}+m_{t}$, so the branching ratio for $\tilde{g} \rightarrow \tilde{t}_{1} t$ decays is $100 \%$. Stops are produced via $\tilde{g} \tilde{g}$ and $\tilde{t}_{1} \tilde{t}_{1}$ and are assumed to decay exclusively via $\tilde{t}_{1} \rightarrow b \tilde{\chi}_{1}^{ \pm}$. The neutralino mass is set to $60 \mathrm{GeV}$, the chargino mass to $120 \mathrm{GeV}$ and the latter is assumed to decay through a virtual $W$ boson. Exclusion limits are presented in the $\left(m_{\tilde{g}}, m_{\tilde{t}_{1}}\right)$ plane.

Gtt model Simplified scenarios, where $\tilde{t}_{1}$ is the lightest squark but $m_{\tilde{g}}<m_{\tilde{t}_{1}}$. Pair production of gluinos is the only process taken into account since the mass of all other sparticles apart from the $\tilde{\chi}_{1}^{0}$ are above the $\mathrm{TeV}$ scale. A three-body decay via off-shell stop is assumed for the gluino, yielding a $100 \%$ BR for the decay $\tilde{g} \rightarrow t \bar{t} \tilde{\chi}_{1}^{0}$. The stop mass has no impact on the kinematics of the decay and the exclusion limits are presented in the $\left(m_{\tilde{g}}, m_{\tilde{\chi}_{1}^{0}}\right)$ plane.

The SR4 regions are mostly sensitive to the SUSY models where sbottom production dominates, whilst the SR6 

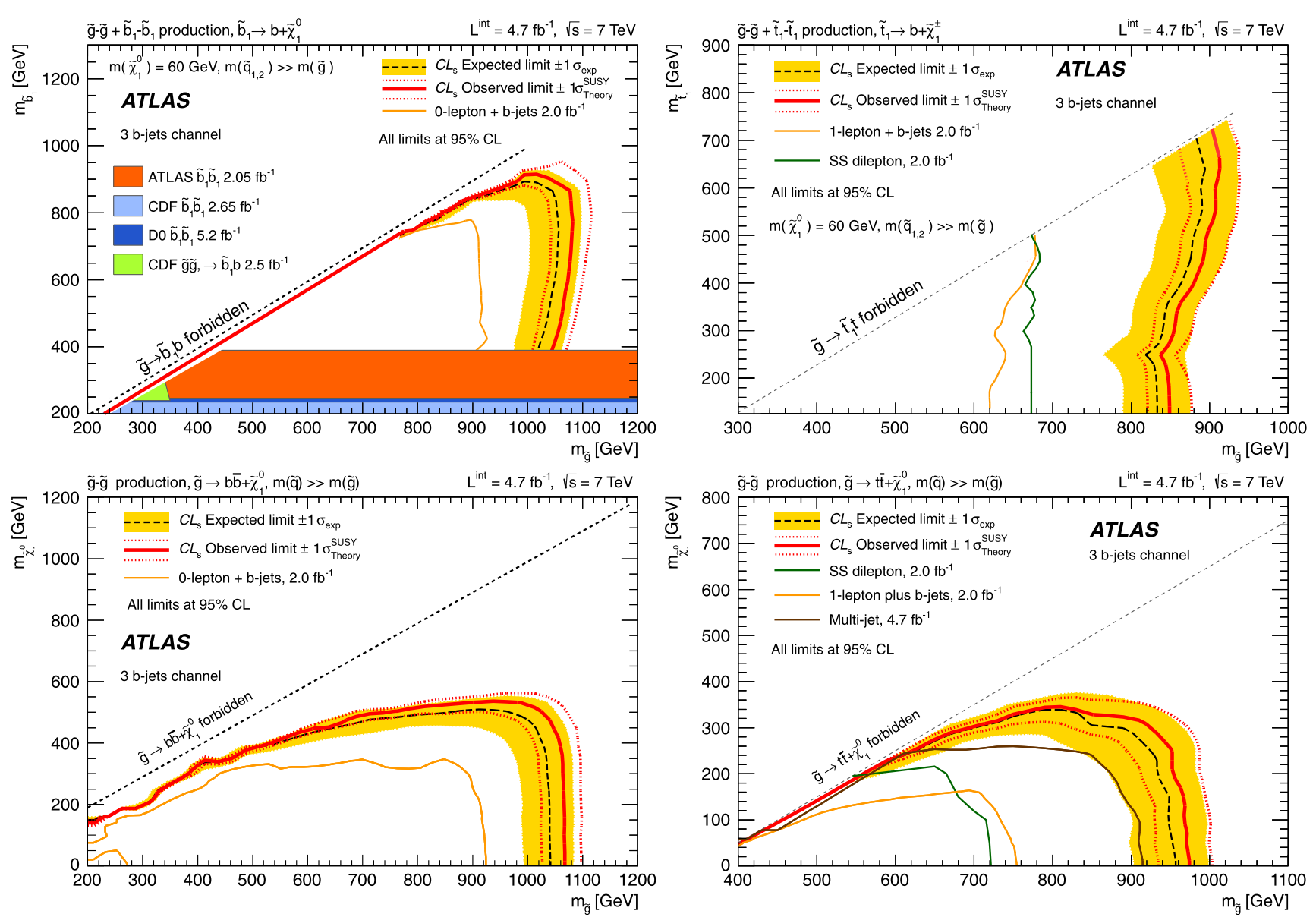

Fig. 2 Exclusion limits in the $\left(m_{\tilde{g}}, m_{\tilde{b}_{1}}\right)$ plane for the gluino-sbottom model (top left), in the $\left(m_{\tilde{g}}, m_{\tilde{t}_{1}}\right)$ plane for the gluino-stop model (top right $)$ and in the $\left(m_{\tilde{g}}, m_{\tilde{\chi}_{1}^{0}}\right)$ plane for the Gbb (bottom left) and Gtt (bottom right) models. The dashed black and solid bold red lines show the $95 \%$ CL expected and observed limits, respectively, including all uncertainties except the theoretical signal cross-section uncertainty. The

shaded (yellow) bands around the expected limits show the impact of the experimental uncertainties while the dotted red lines show the impact on the observed limit of the variation of the nominal signal cross section by $1 \sigma$ theoretical uncertainty. Also shown for reference are the previous CDF [44, 45], D0 [46] and ATLAS [19, 42, 47, 48] analyses (Color figure online)

regions are used to set exclusion limits in models characterized by on-shell or off-shell stop production, where topenriched final states are expected. The signal region with the best expected sensitivity at each point in the parameter space is used to derive the limits at $95 \%$ CL. Signal cross sections are calculated to next-to-leading order in the strong coupling constant, including the resummation of soft gluon emission at next-to-leading-logarithmic accuracy (NLO + NLL) [49-53]. The nominal cross section and the uncertainty $\sigma_{\text {Theory }}^{\text {SUSY }}$ are taken from an envelope of crosssection predictions using different PDF sets and factorization and renormalization scales, as described in Ref. [54]. All detector-related systematic uncertainties are treated as fully correlated between signal and backgrounds. In the $G b b$ scenario, the impact of initial-state radiation (ISR) is expected to be large in the region with low $m_{\tilde{g}}-m_{\tilde{\chi}_{1}^{0}}$ due to the small signal acceptance. Therefore, an uncertainty on the modeling of ISR is assessed by comparing the signal accep-

tance obtained with the Herwig++ samples to the one obtained with dedicated MADGRAPH samples generated with additional jets. This uncertainty varies from $4 \%$ to $35 \%$ as a function of $m_{\tilde{g}}-m_{\tilde{\chi}_{1}^{0}}$ and is included in the $\pm 1 \sigma_{\text {Theory }}^{\text {SUSY }}$ band.

The expected and observed $95 \%$ CL exclusion limits in the four models considered above are shown in Fig. 2. In the gluino-sbottom model, gluino masses below $1000 \mathrm{GeV}$ are excluded for sbottom masses up to about $870 \mathrm{GeV}$ using the most conservative $-1 \sigma_{\text {Theory }}^{\text {SUSY }}$ hypothesis. This extends by approximatively $100 \mathrm{GeV}$ the limits derived in the same scenario by the previous ATLAS analysis performed with $2 \mathrm{fb}^{-1}$ [19] and is complementary to the ATLAS search for direct sbottom pair production, also carried out with $2 \mathrm{fb}^{-1}$ [47]. The exclusion is less stringent in the region with low $m_{\tilde{g}}-m_{\tilde{b}_{1}}$, where softer jets are expected. Because of the kinematic cuts applied, the limits depend on the neutralino mass assumption for low mass splitting between the 
sbottom and the neutralino as shown for the $G b b$ model where gluino masses below $1020 \mathrm{GeV}$ are excluded for neutralino masses up to about $400 \mathrm{GeV}$, improving the previous ATLAS limits [19] by approximatively $100 \mathrm{GeV}$. In the gluino-stop model, gluino masses below $820 \mathrm{GeV}$ are excluded for stop masses up to $640 \mathrm{GeV}$, extending the previous ATLAS limits [19, 42] by approximatively $150 \mathrm{GeV}$. In the $\mathrm{Gtt}$ model, gluino masses below $940 \mathrm{GeV}$ are excluded for $m_{\tilde{\chi}_{1}^{0}}<50 \mathrm{GeV}$ while neutralino masses below $320 \mathrm{GeV}$ are excluded for $m_{\tilde{g}}=800 \mathrm{GeV}$. This search extends the exclusion limits on the gluino mass from the ATLAS multijet analysis carried out with the same data set [48] and from the CMS same-sign dilepton analysis performed with $5 \mathrm{fb}^{-1}[20]$ by approximatively $60 \mathrm{GeV}$ and $130 \mathrm{GeV}$, respectively, for neutralino masses below $100 \mathrm{GeV}$. In the region with low $m_{\tilde{g}}-m_{\tilde{\chi}_{1}^{0}}$, the limits obtained with the CMS analysis are most stringent due to the softer kinematic cuts.

In summary, this letter presents results from a search for top and bottom squarks in the decay of gluino pairs produced in $p p$ collisions at $\sqrt{s}=7 \mathrm{TeV}$, based on $4.7 \mathrm{fb}^{-1}$ of ATLAS data. The events are selected with large $E_{\mathrm{T}}^{\text {miss }}$, four or six jets and at least three jets originating from $b$-quarks in the final state. The results are in agreement with the SM background prediction and translate into $95 \% \mathrm{CL}$ upper limits on excluded masses for a variety of SUSY benchmark scenarios. Gluino masses up to $1 \mathrm{TeV}$ are excluded, depending on the model, which significantly extends the previous results.

Acknowledgements We thank CERN for the very successful operation of the LHC, as well as the support staff from our institutions without whom ATLAS could not be operated efficiently. We acknowledge the support of ANPCyT, Argentina; YerPhI, Armenia; ARC, Australia; BMWF, Austria; ANAS, Azerbaijan; SSTC, Belarus; CNPq and FAPESP, Brazil; NSERC, NRC and CFI, Canada; CERN; CONICYT, Chile; CAS, MOST and NSFC, China; COLCIENCIAS, Colombia; MSMT CR, MPO CR and VSC CR, Czech Republic; DNRF, DNSRC and Lundbeck Foundation, Denmark; ARTEMIS, European Union; IN2P3-CNRS, CEA-DSM/IRFU, France; GNAS, Georgia; BMBF, DFG, HGF, MPG and AvH Foundation, Germany; GSRT, Greece; ISF, MINERVA, GIF, DIP and Benoziyo Center, Israel; INFN, Italy; MEXT and JSPS, Japan; CNRST, Morocco; FOM and NWO, Netherlands; RCN, Norway; MNiSW, Poland; GRICES and FCT, Portugal; MERYS (MECTS), Romania; MES of Russia and ROSATOM, Russian Federation; JINR; MSTD, Serbia; MSSR, Slovakia; ARRS and MVZT, Slovenia; DST/NRF, South Africa; MICINN, Spain; SRC and Wallenberg Foundation, Sweden; SER, SNSF and Cantons of Bern and Geneva, Switzerland; NSC, Taiwan; TAEK, Turkey; STFC, the Royal Society and Leverhulme Trust, United Kingdom; DOE and NSF, United States of America. The crucial computing support from all WLCG partners is acknowledged gratefully, in particular from CERN and the ATLAS Tier-1 facilities at TRIUMF (Canada), NDGF (Denmark, Norway, Sweden), CC-IN2P3 (France), KIT/GridKA (Germany), INFNCNAF (Italy), NL-T1 (Netherlands), PIC (Spain), ASGC (Taiwan), RAL (UK) and BNL (USA) and in the Tier-2 facilities worldwide.

Open Access This article is distributed under the terms of the Creative Commons Attribution License which permits any use, distribution, and reproduction in any medium, provided the original author(s) and the source are credited.

\section{References}

1. H. Miyazawa, Prog. Theor. Phys. 36(6), 1266 (1966)

2. P. Ramond, Phys. Rev. D 3, 2415 (1971)

3. Y. Golfand, E. Likhtman, JETP Lett. 13, 323 (1971)

4. A. Neveu, J.H. Schwarz, Nucl. Phys. B 31, 86 (1971)

5. A. Neveu, J.H. Schwarz, Phys. Rev. D 4, 1109 (1971)

6. J. Gervais, B. Sakita, Nucl. Phys. B 34, 632 (1971)

7. D. Volkov, V. Akulov, Phys. Lett. B 46, 109 (1973)

8. J. Wess, B. Zumino, Phys. Lett. B 49, 52 (1974)

9. J. Wess, B. Zumino, Nucl. Phys. B 70, 39 (1974)

10. S. Weinberg, Phys. Rev. D 13, 974 (1976)

11. E. Gildener, Phys. Rev. D 14, 1667 (1976)

12. S. Weinberg, Phys. Rev. D 19, 1277 (1979)

13. L. Susskind, Phys. Rev. D 20, 2619 (1979)

14. P. Fayet, Phys. Lett. B 64, 159 (1976)

15. P. Fayet, Phys. Lett. B 69, 489 (1977)

16. G.R. Farrar, P. Fayet, Phys. Lett. B 76, 575 (1978)

17. P. Fayet, Phys. Lett. B 84, 416 (1979)

18. S. Dimopoulos, H. Georgi, Nucl. Phys. B 193, 150 (1981)

19. ATLAS Collaboration, Phys. Rev. D 85, 112006 (2012). arXiv:1203.6193 [hep-ex]

20. CMS Collaboration, J. High Energy Phys. 08, 110 (2012)

21. ATLAS Collaboration, J. Instrum. 3, S08003 (2008)

22. M. Mangano et al., J. High Energy Phys. 07, 001 (2003)

23. J. Pumplin et al., J. High Energy Phys. 07, 012 (2002)

24. S. Frixione, B. Webber, arXiv:hep-ph/0601192 (2006)

25. P.M. Nadolsky et al., Phys. Rev. D 78, 013004 (2008)

26. G. Corcella et al., J. High Energy Phys. 01, 010 (2001)

27. G. Corcella et al., arXiv:hep-ph/0210213 (2002)

28. J. Butterworth et al., Z. Phys. C 72, 637 (1996)

29. J. Alwall et al., J. High Energy Phys. 06, 128 (2011)

30. T. Sjöstrand et al., J. High Energy Phys. 0605, 026 (2006)

31. M. Bahr et al., Eur. Phys. J. C 58, 639 (2008)

32. ATLAS Collaboration, Eur. Phys. J. C 70, 823 (2010)

33. S. Agostinelli et al., Nucl. Instrum. Methods Phys. Res. A 506, 250 (2003)

34. M. Cacciari et al., J. High Energy Phys. 04, 063 (2008)

35. M. Cacciari, G. Salam, Phys. Lett. B 641(1), 57 (2006)

36. ATLAS Collaboration, arXiv:1112.6426 [hep-ex] (2011). Submitted to Eur. Phys. J. C

37. ATLAS Collaboration, ATLAS-CONF-2012-043 (2012)

38. ATLAS Collaboration, ATLAS-CONF-2012-039 (2012)

39. ATLAS Collaboration, ATLAS-CONF-2012-040 (2012)

40. ATLAS Collaboration, Eur. Phys. J. C 72, 1909 (2012). arXiv: 1110.3174 [hep-ex]

41. ATLAS Collaboration, arXiv:1208.0949 [hep-ex] (2012). Submitted to Phys. Rev. D

42. ATLAS Collaboration, Phys. Rev. Lett. 108, 241802 (2012). arXiv:1203.5763 [hep-ex]

43. G. Cowan et al., Eur. Phys. J. C 71, 1554 (2011)

44. T. Aaltonen et al. (CDF Collaboration), Phys. Rev. Lett. 102, 221801 (2009)

45. CDF Collaboration, Phys. Rev. Lett. 105, 081802 (2010)

46. D0 Collaboration, Phys. Lett. B 693, 95 (2010)

47. ATLAS Collaboration, Phys. Rev. Lett. 108, 181802 (2012). arXiv:1112.3832 [hep-ex]

48. Z. Zumas (ATLAS Collaboration), J. High Energy Phys. 1207, 167 (2012). arXiv:1206.1760 [hep-ex]

49. W. Beenakker et al., Nucl. Phys. B 492, 51 (1997)

50. A. Kulesza, L. Motyka, Phys. Rev. Lett. 102, 111802 (2009)

51. A. Kulesza, L. Motyka, Phys. Rev. D 80, 095004 (2009)

52. W. Beenakker et al., J. High Energy Phys. 0912, 041 (2009)

53. W. Beenakker et al., Int. J. Mod. Phys. A 26, 2637 (2011)

54. M. Kramer et al., arXiv:1206.2892 [hep-ph] (2012) 


\section{The ATLAS Collaboration}

G. Aad $^{47}$, T. Abajyan ${ }^{20}$, B. Abbott ${ }^{110}$, J. Abdallah ${ }^{11}$, S. Abdel Khalek ${ }^{114}$, A.A. Abdelalim ${ }^{48}$, O. Abdinov ${ }^{10}$, R. Aben ${ }^{104}$, B. Abi $^{111}$, M. Abolins ${ }^{87}$, O.S. AbouZeid ${ }^{157}$, H. Abramowicz ${ }^{152}$, H. Abreu ${ }^{135}$, E. Acerbi ${ }^{88 a, 88 b}$, B.S. Acharya ${ }^{163 a, 163 b}$, L. Adamczyk ${ }^{37}$, D.L. Adams ${ }^{24}$, T.N. Addy ${ }^{55}$, J. Adelman ${ }^{175}$, S. Adomeit ${ }^{97}$, P. Adragna ${ }^{74}$, T. Adye ${ }^{128}$, S. Aefsky ${ }^{22}$, J.A. Aguilar-Saavedra ${ }^{123 b, a}$, M. Agustoni ${ }^{16}$, M. Aharrouche ${ }^{80}$, S.P. Ahlen ${ }^{21}$, F. Ahles ${ }^{47}$, A. Ahmad ${ }^{147}$, M. Ahsan ${ }^{40}$, G. Aielli ${ }^{132 a, 132 b}$, T. Akdogan ${ }^{18 a}$, T.P.A. Åkesson ${ }^{78}$, G. Akimoto ${ }^{154}$, A.V. Akimov ${ }^{93}$, M.S. Alam ${ }^{1}$, M.A. Alam ${ }^{75}$, J. Albert $^{168}$, S. Albrand ${ }^{54}$, M. Aleksa ${ }^{29}$, I.N. Aleksandrov ${ }^{63}$, F. Alessandria ${ }^{88 a}$, C. Alexa ${ }^{25 a}$, G. Alexander ${ }^{152}$, G. Alexan$\mathrm{dre}^{48}$, T. Alexopoulos ${ }^{9}$, M. Alhroob ${ }^{163 a, 163 c}$, M. Aliev ${ }^{15}$, G. Alimonti ${ }^{88 a}$, J. Alison ${ }^{119}$, B.M.M. Allbrooke ${ }^{17}$, P.P. Allport ${ }^{72}$, S.E. Allwood-Spiers ${ }^{52}$, J. Almond ${ }^{81}$, A. Aloisio ${ }^{101 a, 101 b}$, R. Alon ${ }^{171}$, A. Alonso ${ }^{78}$, F. Alonso ${ }^{69}$, B. Alvarez Gonzalez ${ }^{87}$, M.G. Alviggi ${ }^{101 a, 101 b}$, K. Amako ${ }^{64}$, C. Amelung ${ }^{22}$, V.V. Ammosov ${ }^{127, *}$, A. Amorim ${ }^{123 a, b}$, N. Amram ${ }^{152}$, C. Anastopoulos ${ }^{29}$, L.S. Ancu ${ }^{16}$, N. Andari ${ }^{114}$, T. Andeen ${ }^{34}$, C.F. Anders ${ }^{57 b}$, G. Anders ${ }^{57 a}$, K.J. Anderson ${ }^{30}$, A. Andreazza ${ }^{88 a, 88 b}$, V. Andrei ${ }^{57 a}$, X.S. Anduaga ${ }^{69}$, P. Anger ${ }^{43}$, A. Angerami ${ }^{34}$, F. Anghinolfi ${ }^{29}$, A. Anisenkov ${ }^{106}$, N. Anjos ${ }^{123 a}$, A. Annovi ${ }^{46}$, A. Antonaki ${ }^{8}$, M. Antonellii ${ }^{46}$, A. Antonov ${ }^{95}$, J. Antos ${ }^{143 b}$, F. Anulli ${ }^{131 a}$, M. Aoki ${ }^{100}$, S. Aoun ${ }^{82}$, L. Aperio Bella ${ }^{4}$, R. Apolle ${ }^{117, c}$, G. Arabidze $^{87}$, I. Aracena ${ }^{142}$, Y. Arai ${ }^{64}$, A.T.H. Arce ${ }^{44}$, S. Arfaoui ${ }^{147}$, J-F. Arguin ${ }^{14}$, E. Arik ${ }^{18 a, *}$, M. Arik ${ }^{18 a}$, A.J. Armbruster ${ }^{86}$, O. Arnaez ${ }^{80}$, V. Arnal $^{79}$, C. Arnault ${ }^{114}$, A. Artamonov ${ }^{94}$, G. Artoni ${ }^{131 a, 131 b}$, D. Arutinov ${ }^{20}$, S. Asai ${ }^{154}$, R. Asfandiyarov ${ }^{172}$, S. Ask ${ }^{27}$, B. Åsman ${ }^{145 a, 145 b}$, L. Asquith ${ }^{5}$, K. Assamagan ${ }^{24}$, A. Astbury ${ }^{168}$, B. Aubert ${ }^{4}$, E. Auge ${ }^{114}$, K. Augsten ${ }^{126}$, M. Aurousseau $^{144 a}$, G. Avolio ${ }^{162}$, R. Avramidou ${ }^{9}$, D. Axen ${ }^{167}$, G. Azuelos ${ }^{92, d}$, Y. Azuma ${ }^{154}$, M.A. Baak ${ }^{29}$, G. Baccaglioni ${ }^{88 a}$, C. Bacci ${ }^{133 a, 133 b}$, A.M. Bach ${ }^{14}$, H. Bachacou ${ }^{135}$, K. Bachas ${ }^{29}$, M. Backes ${ }^{48}$, M. Backhaus ${ }^{20}$, E. Badescu ${ }^{25 a}$, P. Bagnaia $^{131 \mathrm{a}, 131 b}$, S. Bahinipati ${ }^{2}$, Y. Bai ${ }^{32 a}$, D.C. Bailey ${ }^{157}$, T. Bain ${ }^{157}$, J.T. Baines ${ }^{128}$, O.K. Baker ${ }^{175}$, M.D. Baker ${ }^{24}$, S. Baker ${ }^{76}$, E. Banas $^{38}$, P. Banerjee ${ }^{92}$, Sw. Banerjee ${ }^{172}$, D. Banfi ${ }^{29}$, A. Bangert ${ }^{149}$, V. Bansal ${ }^{168}$, H.S. Bansil ${ }^{17}$, L. Barak ${ }^{171}$, S.P. Bara-

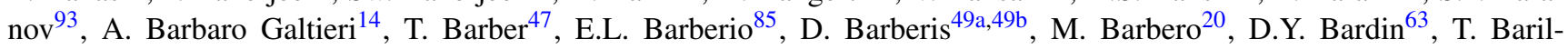
lari $^{98}$, M. Barisonzi ${ }^{174}$, T. Barklow ${ }^{142}$, N. Barlow ${ }^{27}$, B.M. Barnett ${ }^{128}$, R.M. Barnett ${ }^{14}$, A. Baroncelli ${ }^{133 a}$, G. Barone ${ }^{48}$, A.J. Barr ${ }^{117}$, F. Barreiro ${ }^{79}$, J. Barreiro Guimarães da Costa $^{56}$, P. Barrillon ${ }^{114}$, R. Bartoldus ${ }^{142}$, A.E. Barton ${ }^{70}$, V. Bartsch ${ }^{148}$, R.L. Bates ${ }^{52}$, L. Batkova ${ }^{143 a}$, J.R. Batley ${ }^{27}$, A. Battaglia ${ }^{16}$, M. Battistin ${ }^{29}$, F. Bauer ${ }^{135}$, H.S. Bawa ${ }^{142, e}$, S. Beale ${ }^{97}$, T. Beau ${ }^{77}$, P.H. Beauchemin ${ }^{160}$, R. Beccherle ${ }^{49 a}$, P. Bechtle ${ }^{20}$, H.P. Beck ${ }^{16}$, A.K. Becker ${ }^{174}$, S. Becker ${ }^{97}$, M. Beckingham $^{137}$, K.H. Becks ${ }^{174}$, A.J. Beddall ${ }^{18 c}$, A. Beddall ${ }^{18 c}$, S. Bedikian ${ }^{175}$, V.A. Bednyakov ${ }^{63}$, C.P. Bee ${ }^{82}$, L.J. Beemster ${ }^{104}$, M. Begel ${ }^{24}$, S. Behar Harpaz ${ }^{151}$, M. Beimforde ${ }^{98}$, C. Belanger-Champagne ${ }^{84}$, P.J. Bell ${ }^{48}$, W.H. Bell ${ }^{48}$, G. Bella ${ }^{152}$, L. Bellagamba ${ }^{19 a}$, F. Bellina ${ }^{29}$, M. Bellomo ${ }^{29}$, A. Belloni ${ }^{56}$, O. Beloborodova ${ }^{106, f}$, K. Belotskiy ${ }^{95}$, O. Beltramello ${ }^{29}$, O. Benary $^{152}$, D. Benchekroun ${ }^{134 a}$, K. Bendtz ${ }^{145 a, 145 b}$, N. Benekos ${ }^{164}$, Y. Benhammou ${ }^{152}$, E. Benhar Noccioli ${ }^{48}$, J.A. Benitez Garcia $^{158 b}$, D.P. Benjamin ${ }^{44}$, M. Benoit ${ }^{114}$, J.R. Bensinger ${ }^{22}$, K. Benslama ${ }^{129}$, S. Bentvelsen ${ }^{104}$, D. Berge ${ }^{29}$, E. Bergeaas Kuutmann $^{41}$, N. Berger ${ }^{4}$, F. Berghaus ${ }^{168}$, E. Berglund ${ }^{104}$, J. Beringer ${ }^{14}$, P. Bernat ${ }^{76}$, R. Bernhard ${ }^{47}$, C. Bernius ${ }^{24}$, T. Berry ${ }^{75}$, C. Bertella ${ }^{82}$, A. Bertin ${ }^{19 a, 19 b}$, F. Bertolucci ${ }^{121 a, 121 b}$, M.I. Besana ${ }^{88 a, 88 b}$, G.J. Besjes ${ }^{103}$, N. Besson ${ }^{135}$, S. Bethke ${ }^{98}$, W. Bhimji ${ }^{45}$, R.M. Bianchi ${ }^{29}$, M. Bianco ${ }^{71 \mathrm{a}, 71 \mathrm{~b}}$, O. Biebel ${ }^{97}$, S.P. Bieniek ${ }^{76}$, K. Bierwagen ${ }^{53}$, J. Biesiada ${ }^{14}$, M. Biglietti $^{133 a}$, H. Bilokon ${ }^{46}$, M. Bindi ${ }^{19 a, 19 b}$, S. Binet ${ }^{114}$, A. Bingul ${ }^{18 c}$, C. Bini ${ }^{131 a, 131 b}$, C. Biscarat ${ }^{177}$, U. Bitenc ${ }^{47}$, K.M. Black ${ }^{21}$, R.E. Blair ${ }^{5}$, J.-B. Blanchard ${ }^{135}$, G. Blanchot ${ }^{29}$, T. Blazek ${ }^{143 a}$, C. Blocker ${ }^{22}$, J. Blocki ${ }^{38}$, A. Blondel ${ }^{48}$, W. Blum ${ }^{80}$, U. Blumenschein $^{53}$, G.J. Bobbink ${ }^{104}$, V.B. Bobrovnikov ${ }^{106}$, S.S. Bocchetta ${ }^{78}$, A. Bocci ${ }^{44}$, C.R. Boddy ${ }^{117}$, M. Boehler ${ }^{47}$, J. Boek ${ }^{174}$, N. Boelaert ${ }^{35}$, J.A. Bogaerts 29 , A. Bogdanchikov ${ }^{106}$, A. Bogouch ${ }^{89, *}$, C. Bohm ${ }^{145 a}$, J. Bohm ${ }^{124}$, V. Boisvert ${ }^{75}$, T. Bold ${ }^{37}$, V. Boldea ${ }^{25 a}$, N.M. Bolnet ${ }^{135}$, M. Bomben ${ }^{77}$, M. Bona ${ }^{74}$, M. Boonekamp ${ }^{135}$, C.N. Booth ${ }^{138}$, S. Bordoni ${ }^{77}$, C. Borer ${ }^{16}$, A. Borisov ${ }^{127}$, G. Borissov ${ }^{70}$, I. Borjanovic ${ }^{12 a}$, M. Borri ${ }^{81}$, S. Borroni ${ }^{86}$, V. Bortolotto ${ }^{133 a, 133 b}$, K. Bos ${ }^{104}$, D. Boscherini ${ }^{19 a}$, M. Bosman ${ }^{11}$, H. Boterenbrood ${ }^{104}$, J. Bouchami ${ }^{92}$, J. Boudreau ${ }^{122}$, E.V. Bouhova-Thacker ${ }^{70}$, D. Boumediene ${ }^{33}$, C. Bourdarios $^{114}$, N. Bousson ${ }^{82}$, A. Boveia ${ }^{30}$, J. Boyd ${ }^{29}$, I.R. Boyko ${ }^{63}$, I. Bozovic-Jelisavcic ${ }^{12 b}$, J. Bracinik ${ }^{17}$, P. Branchini1 ${ }^{133 a}$, A. Brandt ${ }^{7}$, G. Brandt ${ }^{117}$, O. Brandt ${ }^{53}$, U. Bratzler ${ }^{155}$, B. Brau ${ }^{83}$, J.E. Brau ${ }^{113}$, H.M. Braun ${ }^{174, *}$, S.F. Brazzale ${ }^{163 a, 163 c}$, B. Brelier ${ }^{157}$, J. Bremer ${ }^{29}$, K. Brendlinger ${ }^{119}$, R. Brenner ${ }^{165}$, S. Bressler ${ }^{171}$, D. Britton ${ }^{52}$, F.M. Brochu ${ }^{27}$, I. Brock ${ }^{20}$, R. Brock ${ }^{87}$, F. Broggi ${ }^{88 a}$, C. Bromberg ${ }^{87}$, J. Bronner ${ }^{98}$, G. Brooijmans ${ }^{34}$, T. Brooks ${ }^{75}$, W.K. Brooks ${ }^{31 b}$, G. Brown ${ }^{81}$, H. Brown ${ }^{7}$, P.A. Bruckman de Renstrom ${ }^{38}$, D. Bruncko ${ }^{143 b}$, R. Bruneliere ${ }^{47}$, S. Brunet ${ }^{59}$, A. Bruni ${ }^{19 a}$, G. Bruni ${ }^{19 a}, \mathrm{M} . \mathrm{Br}^{4}$ uschi $^{19 a}$, T. Buanes ${ }^{13}$, Q. Buat ${ }^{54}$, F. Bucci ${ }^{48}$, J. Buchanan ${ }^{117}$, P. Buchholz ${ }^{140}$, R.M. Buckingham ${ }^{117}$, A.G. Buckley ${ }^{45}$, S.I. Buda ${ }^{25 a}$, I.A. Budagov ${ }^{63}$, B. Budick ${ }^{107}$, V. Büscher ${ }^{80}$, L. Bugge ${ }^{116}$, O. Bulekov ${ }^{95}$, A.C. Bundock ${ }^{72}$, M. Bunse ${ }^{42}$, T. Buran ${ }^{116}$, H. Burckhart ${ }^{29}$, S. Burdin ${ }^{72}$, T. Burgess ${ }^{13}$, S. Burke ${ }^{128}$, E. Busato ${ }^{33}$, P. Bussey ${ }^{52}$, C.P. Buszello ${ }^{165}$, B. Butler $^{142}$, J.M. Butler ${ }^{21}$, C.M. Buttar ${ }^{52}$, J.M. Butterworth ${ }^{76}$, W. Buttinger ${ }^{27}$, S. Cabrera Urbán ${ }^{166}$, D. Caforio ${ }^{19 a, 19 b}$, O. Cakir ${ }^{3 a}$, P. Calafiura ${ }^{14}$, G. Calderini ${ }^{77}$, P. Calfayan ${ }^{97}$, R. Calkins ${ }^{105}$, L.P. Caloba ${ }^{23 a}$, R. Caloi ${ }^{131 a, 131 b}$, D. Calvet ${ }^{33}$, S. Calvet ${ }^{33}$, R. Camacho Toro ${ }^{33}$, P. Camarri1 ${ }^{132 a, 132 b}$, D. Cameron ${ }^{116}$, L.M. Caminada ${ }^{14}$, S. Campana ${ }^{29}$, M. Campanelli ${ }^{76}$, V. Canale ${ }^{101 a, 101 b}$, F. Canelli ${ }^{30, g}$, A. Canepa ${ }^{158 a}$, J. Cantero ${ }^{79}$, R. Cantrill ${ }^{75}$, L. Capasso ${ }^{101 a, 101 b}$, M.D.M. Capeans Garrido ${ }^{29}$, I. Caprini ${ }^{25 a}$, 
M. Caprini ${ }^{25 a}$, D. Capriotti ${ }^{98}$, M. Capua ${ }^{36 a, 36 b}$, R. Caputo $^{80}$, R. Cardarelli ${ }^{132 a}$, T. Carli ${ }^{29}$, G. Carlino ${ }^{101 a}$, L. Carminati $^{88 a, 88 b}$, B. Caron ${ }^{84}$, S. Caron ${ }^{103}$, E. Carquin ${ }^{31 b}$, G.D. Carrillo Montoya ${ }^{172}$, A.A. Carter ${ }^{74}$, J.R. Carter ${ }^{27}$, J. Carvalho ${ }^{123 a, h}$, D. Casadei ${ }^{107}$, M.P. Casado ${ }^{11}$, M. Cascella ${ }^{121 a, 121 b}$, C. Caso ${ }^{49 a, 49 b, *}$, A.M. Castaneda Hernandez ${ }^{172, i}$, E. CastanedaMiranda $^{172}$, V. Castillo Gimenez ${ }^{166}$, N.F. Castro ${ }^{123 a}$, G. Cataldi ${ }^{71 a}$, P. Catastini ${ }^{56}$, A. Catinaccio ${ }^{29}$, J.R. Catmore ${ }^{29}$, A. Cattai $^{29}$, G. Cattani ${ }^{132 a, 132 b}$, S. Caughron ${ }^{87}$, P. Cavalleri ${ }^{77}$, D. Cavalli ${ }^{88 a}$, M. Cavalli-Sforza ${ }^{11}$, V. Cavasinni1 ${ }^{121 a, 121 b}$, F. Ceradini $^{133 a, 133 b}$, A.S. Cerqueira ${ }^{23 b}$, A. Cerri ${ }^{29}$, L. Cerrito ${ }^{74}$, F. Cerutti ${ }^{46}$, S.A. Cetin ${ }^{18 b}$, A. Chafaq ${ }^{134 a}$, D. Chakraborty ${ }^{105}$, I. Chalupkova ${ }^{125}$, K. Chan ${ }^{2}$, B. Chapleau ${ }^{84}$, J.D. Chapman ${ }^{27}$, J.W. Chapman ${ }^{86}$, E. Chareyre ${ }^{77}$, D.G. Charlton ${ }^{17}$, V. Chavda ${ }^{81}$, C.A. Chavez Barajas ${ }^{29}$, S. Cheatham ${ }^{84}$, S. Chekanov ${ }^{5}$, S.V. Chekulaev ${ }^{158 a}$, G.A. Chelkov ${ }^{63}$, M.A. Chelstowska ${ }^{103}$, C. $\mathrm{Chen}^{62}$, H. Chen ${ }^{24}$, S. Chen ${ }^{32 c}$, X. Chen ${ }^{172}$, Y. Chen $^{34}$, A. Cheplakov ${ }^{63}$, R. Cherkaoui El Moursli ${ }^{134 e}$, V. Cherny-

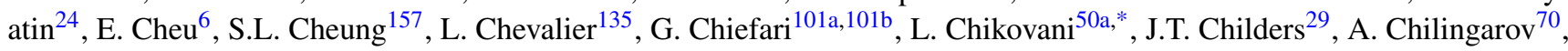
G. Chiodini ${ }^{71 a}$, A.S. Chisholm ${ }^{17}$, R.T. Chislett ${ }^{76}$, A. Chitan ${ }^{25 a}$, M.V. Chizhov ${ }^{63}$, G. Choudalakis ${ }^{30}$, S. Chouridou ${ }^{136}$, I.A. Christidi ${ }^{76}$, A. Christov ${ }^{47}$, D. Chromek-Burckhart ${ }^{29}$, M.L. Chu ${ }^{150}$, J. Chudoba ${ }^{124}$, G. Ciapetti ${ }^{131 a, 131 b}$, A.K. Ciftci ${ }^{3 a}$,

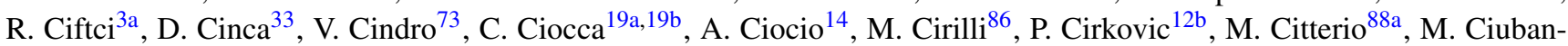
$\mathrm{can}^{25 a}$, A. Clark ${ }^{48}$, P.J. Clark ${ }^{45}$, R.N. Clarke ${ }^{14}$, W. Cleland ${ }^{122}$, J.C. Clemens ${ }^{82}$, B. Clement ${ }^{54}$, C. Clement ${ }^{145 a, 145 b}$, Y. Coadou ${ }^{82}$, M. Cobal ${ }^{163 a, 163 c}$, A. Coccaro ${ }^{137}$, J. Cochran ${ }^{62}$, J.G. Cogan ${ }^{142}$, J. Coggeshall ${ }^{164}$, E. Cogneras ${ }^{177}$, J. Colas $^{4}$, S. Cole ${ }^{105}$, A.P. Colijn ${ }^{104}$, N.J. Collins ${ }^{17}$, C. Collins-Tooth ${ }^{52}$, J. Collot ${ }^{54}$, T. Colombo ${ }^{118 a, 118 b}$, G. Colon ${ }^{83}$, P. Conde Muiño $^{123 a}$, E. Coniavitis ${ }^{117}$, M.C. Conidi ${ }^{11}$, S.M. Consonni ${ }^{88 a, 88 b}$, V. Consorti ${ }^{47}$, S. Constantinescu ${ }^{25 a}$, C. Conta ${ }^{118 a, 118 b}$, G. Conti ${ }^{56}$, F. Conventi ${ }^{101 a, j}$, M. Cooke ${ }^{14}$, B.D. Cooper $^{76}$, A.M. Cooper-Sarkar ${ }^{117}$, K. Copic ${ }^{14}$, T. Cornelissen ${ }^{174}$, M. Corradi $^{19 a}$, F. Corriveau ${ }^{84, k}$, A. Cortes-Gonzalez ${ }^{164}$, G. Cortiana ${ }^{98}$, G. Costa ${ }^{88 a}$, M.J. Costa ${ }^{166}$, D. Costanzo ${ }^{138}$, T. Costin ${ }^{30}$, D. Côté ${ }^{29}$, L. Courneyea ${ }^{168}$, G. Cowan ${ }^{75}$, C. Cowden $^{27}$, B.E. Cox $^{81}$, K. Cranmer $^{107}$, F. Crescioli ${ }^{121 a, 121 b}$, M. Cristinziani ${ }^{20}$, G. Crosetti ${ }^{36 a, 36 b}$, S. Crépé-Renaudin ${ }^{54}$, C.-M. Cuciuc ${ }^{25 a}$, C. Cuenca Almenarr ${ }^{175}$, T. Cuhadar Donszelmann ${ }^{138}, \mathrm{M}^{\mathrm{C}} \mathrm{Cu}-$ ratolo $^{46}$, C.J. Curtis ${ }^{17}$, C. Cuthbert ${ }^{149}$, P. Cwetanski ${ }^{59}$, H. Czirr ${ }^{140}$, P. Czodrowski ${ }^{43}$, Z. Czyczula ${ }^{175}$, S. D'Auria ${ }^{52}$, M. D’Onofrio ${ }^{72}$, A. D’Orazio ${ }^{131 a, 131 b}$, M.J. Da Cunha Sargedas De Sousa ${ }^{123 a}$, C. Da Via ${ }^{81}$, W. Dabrowski ${ }^{37}$, A. Dafinca ${ }^{117}$, T. Dai ${ }^{86}$, C. Dallapiccola ${ }^{83}$, M. Dam ${ }^{35}$, M. Dameri ${ }^{49 a} 49 \mathrm{~b}$, D.S. Damiani ${ }^{136}$, H.O. Danielsson ${ }^{29}$, V. Dao ${ }^{48}$, G. Darbo ${ }^{49 a}$, G.L. Darlea ${ }^{25 b}$, J.A. Dassoulas ${ }^{41}$, W. Davey ${ }^{20}$, T. Davidek ${ }^{125}$, N. Davidson ${ }^{85}$, R. Davidson ${ }^{70}$, E. Davies ${ }^{117, c}$, M. Davies ${ }^{92}$, O. Davignon ${ }^{77}$, A.R. Davison ${ }^{76}$, Y. Davygora ${ }^{57 a}$, E. Dawe ${ }^{141}$, I. Dawson ${ }^{138}$, R.K. Daya-Ishmukhametova ${ }^{22}$, K. De ${ }^{7}$, R. de Asmundis ${ }^{101 a}$, S. De Castro ${ }^{19 a}, 19 b$, S. De $\mathrm{Cecco}^{77}$, J. de Graat ${ }^{97}$, N. De Groot ${ }^{103}$, P. de Jong ${ }^{104}$, C. De La Taille ${ }^{114}$, H. De la Torre ${ }^{79}$, F. De Lorenzi ${ }^{62}$, L. de Mora $^{70}$, L. De Nooij ${ }^{104}$, D. De Pedis ${ }^{131 a}$, A. De Salvo ${ }^{131 a}$, U. De Sanctis $^{163 a, 163 c}$, A. De Santo ${ }^{148}$, J.B. De Vivie De Regie ${ }^{114}$, G. De Zorzi ${ }^{131 a, 131 b}$, W.J. Dearnaley ${ }^{70}$, R. Debbe ${ }^{24}$, C. Debenedetti ${ }^{45}$, B. Dechenaux ${ }^{54}$, D.V. Dedovich ${ }^{63}$, J. Degenhardt ${ }^{119}$, C. Del Papa ${ }^{163 a, 163 c}$, J. Del Peso ${ }^{79}$, T. Del Prete ${ }^{121 a, 121 b}$, T. Delemontex $^{54}$, M. Deliyergiyev ${ }^{73}$, A. Dell'Acqua ${ }^{29}$, L. Dell'Asta ${ }^{21}$, M. Della Pietra ${ }^{101 a, j}$, D. della Volpe ${ }^{101 a, 101 b}$, M. Delmastro $^{4}$, P.A. Delsart ${ }^{54}$, C. Deluca ${ }^{104}$, S. Demers ${ }^{175}$, M. Demichev ${ }^{63}$, B. Demirkoz ${ }^{11,1}$, J. Deng ${ }^{162}$, S.P. Denisov ${ }^{127}$, D. Derendarz ${ }^{38}$, J.E. Derkaoui ${ }^{134 d}$, F. Derue ${ }^{77}$, P. Dervan ${ }^{72}$, K. Desch $^{20}$, E. Devetak ${ }^{147}$, P.O. Deviveiros ${ }^{104}$, A. Dewhurst ${ }^{128}$, B. DeWilde ${ }^{147}$, S. Dhaliwal ${ }^{157}$, R. Dhullipudi ${ }^{24, m}$, A. Di Ciaccio ${ }^{132 a, 132 b}$, L. Di Ciaccio ${ }^{4}$, A. Di Girolamo ${ }^{29}$, B. Di Girolamo $^{29}$, S. Di Luise ${ }^{133 a, 133 b}$, A. Di Mattia ${ }^{172}$, B. Di Micco ${ }^{29}$, R. Di Nardo ${ }^{46}$, A. Di Simone ${ }^{132 a, 132 b}$, R. Di Sipio ${ }^{19 a, 19 b}$, M.A. Diaz ${ }^{31 a}$, E.B. Diehl ${ }^{86}$, J. Dietrich ${ }^{41}$, T.A. Dietzsch ${ }^{57 a}$, S. Diglio ${ }^{85}$, K. Dindar Yagci $^{39}$, J. Dingfelder ${ }^{20}$, F. Dinut ${ }^{25 a}$, C. Dionisi ${ }^{131 a, 131 b}$, P. Dita ${ }^{25 a}$, S. Dita ${ }^{25 a}$, F. Dittus ${ }^{29}$, F. Djama ${ }^{82}$, T. Djobava ${ }^{50 b}$, M.A.B. do Vale ${ }^{23 c}$, A. Do Valle Wemans $^{123 a, n}$, T.K.O. Doan ${ }^{4}$, M. Dobbs ${ }^{84}$, R. Dobinson ${ }^{29, *}$, D. Dobos ${ }^{29}$, E. Dobson ${ }^{29, o}$, J. Dodd $^{34}$, C. Doglioni ${ }^{48}$, T. Doherty ${ }^{52}$, Y. Doi ${ }^{64, *}$, J. Dolejsi ${ }^{125}$, I. Dolenc ${ }^{73}$, Z. Dolezal ${ }^{125}$, B.A. Dolgoshein ${ }^{95, *}$, T. Dohmae ${ }^{154}$, M. Donadelli ${ }^{23 d}$, J. Donini ${ }^{33}$, J. Dopke $^{29}$, A. Doria ${ }^{101 a}$, A. Dos Anjos ${ }^{172}$, A. Dotti ${ }^{121 a, 121 b}$, M.T. Dova ${ }^{69}$, A.D. Doxiadis ${ }^{104}$, A.T. Doyle ${ }^{52}$, M. Dris ${ }^{9}$, J. Dubbert $^{98}$, S. Dube ${ }^{14}$, E. Duchovni ${ }^{171}$, G. Duckeck ${ }^{97}$, A. Dudarev ${ }^{29}$, F. Dudziak ${ }^{62}$, M. Dührssen ${ }^{29}$, I.P. Duerdoth ${ }^{81}$, L. Duflot ${ }^{114}$, M-A. Dufour ${ }^{84}$, L. Duguid ${ }^{75}$, M. Dunford ${ }^{29}$, H. Duran Yildiz $^{3 a}$, R. Duxfield ${ }^{138}$, M. Dwuznik ${ }^{37}$, F. Dydak ${ }^{29}$, M. Düren ${ }^{51}$, J. Ebke ${ }^{97}$, S. Eckweiler ${ }^{80}$, K. Edmonds ${ }^{80}$, W. Edson ${ }^{1}$, C.A. Edwards ${ }^{75}$, N.C. Edwards ${ }^{52}$, W. Ehrenfeld ${ }^{41}$, T. Eifert ${ }^{142}$, G. Eigen ${ }^{13}$, K. Einsweiler ${ }^{14}$, E. Eisenhandler ${ }^{74}$, T. Ekelof ${ }^{165}$, M. El Kacimi ${ }^{134 c}$, M. Ellert ${ }^{165}$, S. Elles ${ }^{4}$, F. Ellinghaus ${ }^{80}$, K. Ellis ${ }^{74}$, N. Ellis $^{29}$, J. Elmsheuser ${ }^{97}$, M. Elsing ${ }^{29}$, D. Emeliyanov ${ }^{128}$, R. Engelmann ${ }^{147}$, A. Engl ${ }^{97}$, B. Epp ${ }^{60}$, J. Erdmann ${ }^{53}$, A. Ereditato ${ }^{16}$, D. Eriksson ${ }^{145 a}$, J. Ernst ${ }^{1}$, M. Ernst ${ }^{24}$, J. Ernwein $^{135}$, D. Errede ${ }^{164}$, S. Errede $^{164}$, E. Ertel ${ }^{80}$, M. Escalier ${ }^{114}$, H. Esch $^{42}$, C. Escobar ${ }^{122}$, X. Espinal Curull ${ }^{11}$, B. Esposito ${ }^{46}$, F. Etienne ${ }^{82}$, A.I. Etienvre ${ }^{135}$, E. Etzion ${ }^{152}$, D. Evangelakou ${ }^{53}$, H. Evans ${ }^{59}$, L. Fabbri ${ }^{19 a, 19 b}$, C. Fabre ${ }^{29}$, R.M. Fakhrutdinov ${ }^{127}$, S. Falciano ${ }^{131 a}$, Y. Fang ${ }^{172}$, M. Fanti ${ }^{88 a, 88 b}$, A. Farbin ${ }^{7}$, A. Farilla ${ }^{133 a}$, J. Farley ${ }^{147}$, T. Farooque ${ }^{157}$, S. Farrell ${ }^{162}$, S.M. Farrington ${ }^{169}$, P. Farthouat ${ }^{29}$, P. Fassnacht ${ }^{29}$, D. Fassouliotis ${ }^{8}$, B. Fatholahzadeh ${ }^{157}$, A. Favareto ${ }^{88 a, 88 b}$, L. Fayard ${ }^{114}$, S. Fazio ${ }^{36 a, 36 b}$, R. Febbraro ${ }^{33}$, P. Federic ${ }^{143 a}$, O.L. Fedin ${ }^{120}$, W. Fedorko $^{87}$, M. Fehling-Kaschek ${ }^{47}$, L. Feligioni ${ }^{82}$, D. Fellmann ${ }^{5}$, C. Feng ${ }^{32 d}$, E.J. Feng 5 , A.B. Fenyuk ${ }^{127}$, J. Ferencei ${ }^{143 b}$, W. Fernando ${ }^{5}$, S. Ferrag ${ }^{52}$, J. Ferrando ${ }^{52}$, V. Ferrara ${ }^{41}$, A. Ferrari ${ }^{165}$, P. Ferrari ${ }^{104}$, R. Ferrari ${ }^{118 a}$, D.E. Ferreira de Lima $^{52}$, A. Ferrer ${ }^{166}$, D. Ferrere ${ }^{48}$, C. Ferretti ${ }^{86}$, A. Ferretto Parodi ${ }^{49 a, 49 b}$, M. Fiascaris ${ }^{30}$, F. Fiedler ${ }^{80}$, A. Filipčič ${ }^{73}$, F. Filthaut ${ }^{103}$, 
M. Fincke-Keeler ${ }^{168}$, M.C.N. Fiolhais ${ }^{123 a, h}$, L. Fiorini ${ }^{166}$, A. Firan ${ }^{39}$, G. Fischer ${ }^{41}$, M.J. Fisher ${ }^{108}$, M. Flechl ${ }^{47}$, I. Fleck $^{140}$, J. Fleckner ${ }^{80}$, P. Fleischmann ${ }^{173}$, S. Fleischmann ${ }^{174}$, T. Flick ${ }^{174}$, A. Floderus ${ }^{78}$, L.R. Flores Castillo ${ }^{172}$, M.J. Flowerdew ${ }^{98}$, T. Fonseca Martin ${ }^{16}$, A. Formica ${ }^{135}$, A. Forti ${ }^{81}$, D. Fortin ${ }^{158 a}$, D. Fournier ${ }^{14}$, H. Fox ${ }^{70}$, P. Francavilla ${ }^{11}$, M. Franchini $^{19 a, 19 b}$, S. Franchino ${ }^{118 a, 118 b}$, D. Francis ${ }^{29}$, T. Frank ${ }^{171}$, S. Franz ${ }^{29}$, M. Fraternali ${ }^{118 a, 118 b}$, S. Fratina ${ }^{119}$, S.T. French ${ }^{27}$, C. Friedrich ${ }^{41}$, F. Friedrich ${ }^{43}$, R. Froesch ${ }^{29}$, D. Froidevaux ${ }^{29}$, J.A. Frost ${ }^{27}$, C. Fukunaga ${ }^{155}$, E. Fullana Torregrosa ${ }^{29}$, B.G. Fulsom ${ }^{142}$, J. Fuster ${ }^{166}$, C. Gabaldon ${ }^{29}$, O. Gabizon ${ }^{171}$, T. Gadfort ${ }^{24}$, S. Gadomski ${ }^{48}$, G. Gagliardi ${ }^{49 a}$,49b , P. Gagnon ${ }^{59}$, C. Galea ${ }^{97}$, E.J. Gallas ${ }^{117}$, V. Gallo ${ }^{16}$, B.J. Gallop ${ }^{128}$, P. Gallus ${ }^{124}$, K.K. Gan ${ }^{108}$, Y.S. Gao ${ }^{142, e}$, A. Gaponenko ${ }^{14}$, F. Garberson $^{175}$, M. Garcia-Sciveres ${ }^{14}$, C. García ${ }^{166}$, J.E. García Navarro ${ }^{166}$, R.W. Gardner ${ }^{30}$, N. Garelli ${ }^{29}$, H. Garitaonandia ${ }^{104}$, V. Garonne ${ }^{29}$, C. Gatti ${ }^{46}$, G. Gaudio ${ }^{118 a}$, B. Gaur ${ }^{140}$, L. Gauthier ${ }^{135}$, P. Gauzzi ${ }^{131 a, 131 b}$, I.L. Gavrilenko ${ }^{93}$, C. Gay ${ }^{167}$, G. Gaycken $^{20}$, E.N. Gazis ${ }^{9}$, P. Ge ${ }^{32 d}$, Z. Gecse ${ }^{167}$, C.N.P. Gee ${ }^{128}$, D.A.A. Geerts ${ }^{104}$, Ch. Geich-Gimbel ${ }^{20}$, K. Gellerst-

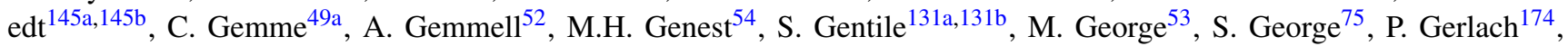
A. Gershon ${ }^{152}$, C. Geweniger ${ }^{57 a}$, H. Ghazlane ${ }^{134 b}$, N. Ghodbane ${ }^{33}$, B. Giacobbe ${ }^{19 a}$, S. Giagu $^{131 a, 131 b}$, V. Giakoumopoulou ${ }^{8}$, V. Giangiobbe ${ }^{11}$, F. Gianotti ${ }^{29}$, B. Gibbard ${ }^{24}$, A. Gibson ${ }^{157}$, S.M. Gibson ${ }^{29}$, D. Gillberg ${ }^{28}$, A.R. Gillman ${ }^{128}$, D.M. Gingrich $^{2, \mathrm{~d}}$, J. Ginzburg ${ }^{152}$, N. Giokaris ${ }^{8}$, M.P. Giordani ${ }^{163 c}$, R. Giordano ${ }^{101 a, 101 b}$, F.M. Giorgi ${ }^{15}$, P. Giovannini ${ }^{98}$, P.F. Gi-

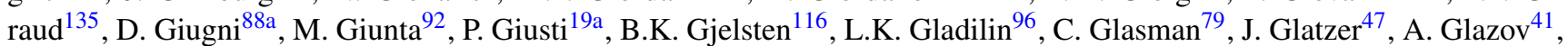
K.W. Glitza ${ }^{174}$, G.L. Glonti ${ }^{63}$, J.R. Goddard ${ }^{74}$, J. Godfrey ${ }^{141}$, J. Godlewski ${ }^{29}$, M. Goebel ${ }^{41}$, T. Göpfert ${ }^{43}$, C. Goeringer ${ }^{80}$, C. Gössling ${ }^{42}$, S. Goldfarb ${ }^{86}$, T. Golling ${ }^{175}$, A. Gomes ${ }^{123 a, b}$, L.S. Gomez Fajardo ${ }^{41}$, R. Gonçalo ${ }^{75}$, J. Goncalves Pinto Firmino Da Costa ${ }^{41}$, L. Gonella ${ }^{20}$, S. Gonzalez ${ }^{172}$, S. González de la Hoz ${ }^{166}$, G. Gonzalez Parra ${ }^{11}$, M.L. Gonzalez Silva ${ }^{26}$, S. Gonzalez-Sevilla ${ }^{48}$, J.J. Goodson ${ }^{147}$, L. Goossens ${ }^{29}$, P.A. Gorbounov ${ }^{94}$, H.A. Gordon ${ }^{24}$, I. Gorelov ${ }^{102}$, G. Gorfine ${ }^{174}$, B. Gorini ${ }^{29}$, E. Gorini ${ }^{71 a}$,71b , A. Gorišek ${ }^{73}$, E. Gornicki ${ }^{38}$, B. Gosdzik ${ }^{41}$, A.T. Goshaw ${ }^{5}$, M. Gosselink ${ }^{104}$, M.I. Gostkin ${ }^{63}$, I. Gough Eschrich ${ }^{162}$, M. Gouighri ${ }^{134 a}$, D. Goujdami ${ }^{134 c}$, M.P. Goulette ${ }^{48}$, A.G. Goussiou ${ }^{137}$, C. Goy ${ }^{4}$, S. Gozpinar ${ }^{22}$, I. Grabowska-Bold ${ }^{37}$, P. Grafström ${ }^{19 a, 19 b}$, K-J. Grahn ${ }^{41}$, F. Grancagnolo ${ }^{71 a}$, S. Grancagnolo ${ }^{15}$, V. Grassi ${ }^{147}$, V. Gratchev ${ }^{120}$, N. $\mathrm{Grau}^{34}$, H.M. Gray ${ }^{29}$, J.A. Gray ${ }^{147}$, E. Graziani ${ }^{133 a}$, O.G. Grebenyuk ${ }^{120}$, T. Greenshaw ${ }^{72}$, Z.D. Greenwood ${ }^{24, \mathrm{~m}}$, K. Gregersen ${ }^{35}$, I.M. Gregor ${ }^{41}$, P. Grenier ${ }^{142}$, J. Griffiths ${ }^{137}$, N. Grigalashvili ${ }^{63}$, A.A. Grillo ${ }^{136}$, S. Grinstein ${ }^{11}$, Y.V. Grishkevich $^{96}$, J.-F. Grivaz ${ }^{114}$, E. Gross ${ }^{171}$, J. Grosse-Knetter ${ }^{53}$, J. Groth-Jensen ${ }^{171}$, K. Grybel ${ }^{140}$, D. Guest ${ }^{175}$, C. Guicheney $^{33}$, S. Guindon ${ }^{53}$, U. Gul ${ }^{52}$, H. Guler ${ }^{84, p}$, J. Gunther ${ }^{124}$, B. Guo ${ }^{157}$, J. Guo ${ }^{34}$, P. Gutierrez ${ }^{110}$, N. Guttman ${ }^{152}$, O. Gutzwiller ${ }^{172}$, C. Guyot ${ }^{135}$, C. Gwenlan ${ }^{117}$, C.B. Gwilliam ${ }^{72}$, A. Haas ${ }^{142}$, S. Haas ${ }^{29}$, C. Haber ${ }^{14}$, H.K. Hadavand ${ }^{39}$,

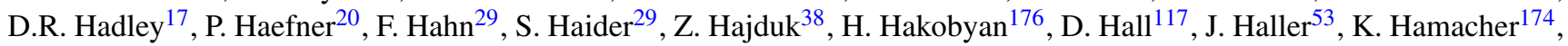
P. Hamal ${ }^{112}$, M. Hamer ${ }^{53}$, A. Hamilton ${ }^{144 b, q}$, S. Hamilton ${ }^{160}$, L. Han ${ }^{32 b}$, K. Hanagaki ${ }^{115}$, K. Hanawa ${ }^{159}$, M. Hance ${ }^{14}$, C. Handel ${ }^{80}$, P. Hanke ${ }^{57 a}$, J.R. Hansen ${ }^{35}$, J.B. Hansen ${ }^{35}$, J.D. Hansen ${ }^{35}$, P.H. Hansen ${ }^{35}$, P. Hansson ${ }^{142}$, K. Hara ${ }^{159}$, G.A. Hare ${ }^{136}$, T. Harenberg ${ }^{174}$, S. Harkusha ${ }^{89}$, D. Harper ${ }^{86}$, R.D. Harrington ${ }^{45}$, O.M. Harris ${ }^{137}$, J. Hartert ${ }^{47}$, F. Hartjes ${ }^{104}$, T. Haruyama ${ }^{64}$, A. Harvey ${ }^{55}$, S. Hasegawa ${ }^{100}$, Y. Hasegawa ${ }^{139}$, S. Hassani ${ }^{135}$, S. Haug ${ }^{16}$, M. Hauschild ${ }^{29}$, R. Hauser ${ }^{87}$, M. Havranek ${ }^{20}$, C.M. Hawkes ${ }^{17}$, R.J. Hawkings ${ }^{29}$, A.D. Hawkins ${ }^{78}$, D. Hawkins ${ }^{162}$, T. Hayakawa ${ }^{65}$, T. Hayashi ${ }^{159}$, D. Hayden ${ }^{75}$, C.P. Hays ${ }^{117}$, H.S. Hayward ${ }^{72}$, S.J. Haywood ${ }^{128}$, M. He ${ }^{32 d}$, S.J. Head ${ }^{17}$, V. Hedberg ${ }^{78}$, L. Heelan ${ }^{7}$, S. Heim ${ }^{87}$, B. Heinemann ${ }^{14}$, S. Heisterkamp ${ }^{35}$, L. Helary ${ }^{21}$, C. Heller ${ }^{97}$, M. Heller ${ }^{29}$, S. Hellman ${ }^{145 a, 145 b}$, D. Hellmich ${ }^{20}$, C. Helsens ${ }^{11}$, R.C.W. Henderson ${ }^{70}$, M. Henke ${ }^{57 a}$, A. Henrichs ${ }^{53}$, A.M. Henriques Correia ${ }^{29}$, S. Henrot-Versille ${ }^{114}$, C. Hensel ${ }^{53}$, T. Hen $\beta^{174}$, C.M. Hernandez ${ }^{7}$, Y. Hernández Jiménez ${ }^{166}$, R. Herrberg ${ }^{15}$, G. Herten ${ }^{47}$, R. Hertenberger ${ }^{97}$, L. Hervas ${ }^{29}$, G.G. Hesketh ${ }^{76}$, N.P. Hessey ${ }^{104}$, E. Higón-Rodriguez ${ }^{166}$, J.C. Hill' ${ }^{27}$, K.H. Hiller ${ }^{41}$, S. Hillert ${ }^{20}$, S.J. Hillier ${ }^{17}$, I. Hinchliffe ${ }^{14}$, E. Hines ${ }^{119}$, M. Hirose ${ }^{115}$, F. Hirsch ${ }^{42}$, D. Hirschbuehl ${ }^{174}$, J. Hobbs ${ }^{147}$, N. Hod ${ }^{152}$, M.C. Hodgkin-

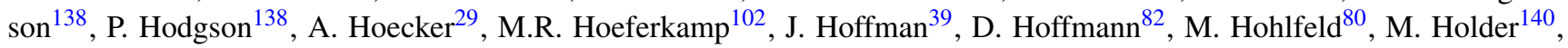
S.O. Holmgren ${ }^{145 a}$, T. Holy ${ }^{126}$, J.L. Holzbauer ${ }^{87}$, T.M. Hong ${ }^{119}$, L. Hooft van Huysduynen ${ }^{107}$, C. Horn ${ }^{142}$, S. Horner ${ }^{47}$, J-Y. Hostachy ${ }^{54}$, S. Hou ${ }^{150}$, A. Hoummada ${ }^{134 a}$, J. Howard ${ }^{117}$, J. Howarth ${ }^{81}$, I. Hristova ${ }^{15}$, J. Hrivnac ${ }^{114}$, T. Hryn'ova ${ }^{4}$, P.J. $\mathrm{Hsu}^{80}$, S.-C. $\mathrm{Hsu}^{14}$, Z. Hubacek ${ }^{126}$, F. Hubaut ${ }^{82}$, F. Huegging ${ }^{20}$, A. Huettmann ${ }^{41}$, T.B. Huffman ${ }^{117}$, E.W. Hughes ${ }^{34}$,

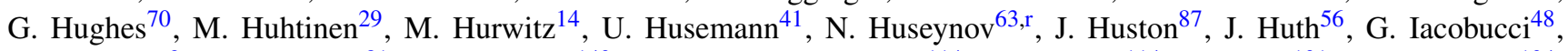
G. Iakovidis $^{9}$, M. Ibbotson ${ }^{81}$, I. Ibragimov ${ }^{140}$, L. Iconomidou-Fayard ${ }^{114}$, J. Idarraga ${ }^{114}$, P. Iengo ${ }^{101 a}$, O. Igonkina ${ }^{104}$,

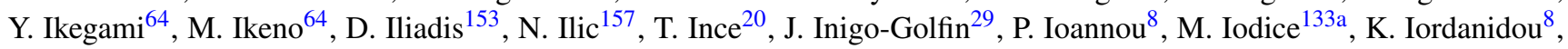
V. Ippolito ${ }^{131 \mathrm{a}, 131 \mathrm{~b}}$, A. Irles Quiles ${ }^{166}$, C. Isaksson ${ }^{165}$, M. Ishino ${ }^{66}$, M. Ishitsuka ${ }^{156}$, R. Ishmukhametov ${ }^{39}$, C. Issever ${ }^{117}$, S. Istin ${ }^{18 a}$, A.V. Ivashin ${ }^{127}$, W. Iwanski ${ }^{38}$, H. Iwasaki ${ }^{64}$, J.M. Izen ${ }^{40}$, V. Izzo ${ }^{101 a}$, B. Jackson ${ }^{119}$, J.N. Jackson ${ }^{72}$, P. Jackson $^{142}$, M.R. Jaekel ${ }^{29}$, V. Jain ${ }^{59}$, K. Jakobs ${ }^{47}$, S. Jakobsen ${ }^{35}$, T. Jakoubek ${ }^{124}$, J. Jakubek ${ }^{126}$, D.K. Jana ${ }^{110}$, E. Jansen ${ }^{76}$, H. Jansen ${ }^{29}$, A. Jantsch ${ }^{98}$, M. Janus ${ }^{47}$, G. Jarlskog ${ }^{78}$, L. Jeanty ${ }^{56}$, I. Jen-La Plante ${ }^{30}$, D. Jennens ${ }^{85}$, P. Jenni ${ }^{29}$, P. Jež ${ }^{35}$, S. Jézéquel ${ }^{4}$, M.K. Jha ${ }^{19 a}$, H. Ji ${ }^{172}$, W. Ji ${ }^{80}$, J. Jia ${ }^{147}$, Y. Jiang ${ }^{32 b}$, M. Jimenez Belenguer ${ }^{41}$, S. Jin ${ }^{32 a}$, O. Jinnouchi ${ }^{156}$, M.D. Joergensen ${ }^{35}$, D. Joffe ${ }^{39}$, M. Johansen ${ }^{145 a, 145 b}$, K.E. Johansson ${ }^{145 a}$, P. Johansson ${ }^{138}$, S. Johnert ${ }^{41}$, K.A. Johns ${ }^{6}$, K. Jon-And ${ }^{145 a, 145 b}$, G. Jones ${ }^{169}$, R.W.L. Jones ${ }^{70}$, T.J. Jones ${ }^{72}$, C. Joram ${ }^{29}$, P.M. Jorge ${ }^{123 a}$, K.D. Joshi ${ }^{81}$, J. Jovicevic ${ }^{146}$, 
T. Jovin ${ }^{12 b}$, X. Ju ${ }^{172}$, C.A. Jung ${ }^{42}$, R.M. Jungst ${ }^{29}$, V. Juranek ${ }^{124}$, P. Jussel ${ }^{60}$, A. Juste Rozas ${ }^{11}$, S. Kabana ${ }^{16}$, M. Kaci ${ }^{166}$, A. Kaczmarska ${ }^{38}$, P. Kadlecik ${ }^{35}$, M. Kado ${ }^{114}$, H. Kagan ${ }^{108}$, M. Kagan ${ }^{56}$, E. Kajomovitz ${ }^{151}$, S. Kalinin ${ }^{174}$, L.V. Kalinovskaya $^{63}$, S. Kama ${ }^{39}$, N. Kanaya ${ }^{154}$, M. Kaneda ${ }^{29}$, S. Kaneti ${ }^{27}$, T. Kanno ${ }^{156}$, V.A. Kantserov ${ }^{95}$, J. Kanzaki ${ }^{64}$, B. Kaplan $^{175}$, A. Kapliy ${ }^{30}$, J. Kaplon ${ }^{29}$, D. Kar ${ }^{52}$, M. Karagounis ${ }^{20}$, K. Karakostas ${ }^{9}$, M. Karnevskiy ${ }^{41}$, V. Kartvelishvili ${ }^{70}$, A.N. Karyukhin ${ }^{127}$, L. Kashif ${ }^{172}$, G. Kasieczka ${ }^{57 b}$, R.D. Kass ${ }^{108}$, A. Kastanas ${ }^{13}$, M. Kataoka ${ }^{4}$, Y. Kataoka ${ }^{154}$, E. Katsoufis $^{9}$, J. Katzy ${ }^{41}$, V. Kaushik ${ }^{6}$, K. Kawagoe ${ }^{68}$, T. Kawamoto ${ }^{154}$, G. Kawamura ${ }^{80}$, M.S. Kay1 ${ }^{104}$, V.A. Kazanin ${ }^{106}$, M.Y. Kazarinov ${ }^{63}$, R. Keeler ${ }^{168}$, R. Kehoe ${ }^{39}$, M. Keil ${ }^{53}$, G.D. Kekelidze ${ }^{63}$, J.S. Keller ${ }^{137}$, M. Kenyon ${ }^{52}$, O. Kepka ${ }^{124}$, N. Kerschen ${ }^{29}$, B.P. Kerševan ${ }^{73}$, S. Kersten ${ }^{174}$, K. Kessoku ${ }^{154}$, J. Keung ${ }^{157}$, F. Khalil-zada ${ }^{10}$, H. Khandanyan ${ }^{164}$, A. Khanov ${ }^{111}$, D. Kharchenko ${ }^{63}$, A. Khodinov ${ }^{95}$, A. Khomich ${ }^{57 a}$, T.J. Khoo ${ }^{27}$, G. Khoriauli ${ }^{20}$, A. Khoroshilov ${ }^{174}$, V. Khovanskiy ${ }^{94}$, E. Khramov ${ }^{63}$, J. Khubua ${ }^{50 b}$, H. Kim ${ }^{145 a, 145 b}$, S.H. Kim ${ }^{159}$, N. Kimura ${ }^{170}$, O. Kind ${ }^{15}$, B.T. King ${ }^{72}$, M. King ${ }^{65}$, R.S.B. King ${ }^{17}$, J. Kirk ${ }^{128}$, A.E. Kiryunin ${ }^{98}$, T. Kishimoto ${ }^{65}$, D. Kisielewska ${ }^{37}$, T. Kitamura ${ }^{65}$, T. Kittelmann ${ }^{122}$, E. Kladiva ${ }^{143 b}$, M. Klein ${ }^{72}$, U. Klein ${ }^{72}$, K. Kleinknecht ${ }^{80}$, M. Klemetti ${ }^{84}$, A. Klier ${ }^{171}$, P. Klimek ${ }^{145 a, 145 b}$, A. Klimentov ${ }^{24}$, R. Klingenberg ${ }^{42}$, J.A. Klinger ${ }^{81}$, E.B. Klinkby ${ }^{35}$, T. Klioutchnikova ${ }^{29}$, P.F. Klok ${ }^{103}$, S. Klous ${ }^{104}$, E.-E. Kluge ${ }^{57 a}$, T. Kluge ${ }^{72}$, P. Kluit ${ }^{104}$, S. Kluth ${ }^{98}$, N.S. Knecht ${ }^{157}$, E. Kneringer ${ }^{60}$, E.B.F.G. Knoops ${ }^{82}$, A. Knue ${ }^{53}$, B.R. Ko ${ }^{44}$, T. Kobayashi ${ }^{154}$, M. Kobel ${ }^{43}$, M. Kociann ${ }^{142}$, P. Kodys ${ }^{125}$, K. Köneke ${ }^{29}$, A.C. König ${ }^{103}$, S. Koenig ${ }^{80}$, L. Köpke ${ }^{80}$, F. Koetsveld ${ }^{103}$, P. Ko-

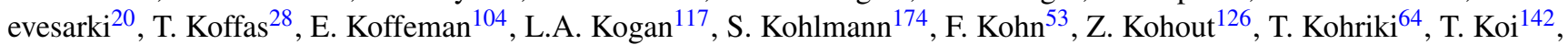
G.M. Kolachev ${ }^{106, *}$, H. Kolanoski ${ }^{15}$, V. Kolesnikov ${ }^{63}$, I. Koletsou ${ }^{88 a}$, J. Koll ${ }^{87}$, M. Kollefrath ${ }^{47}$, A.A. Komar ${ }^{93}$, Y. Komori $^{154}$, T. Kondo ${ }^{64}$, T. Kono ${ }^{41, \mathrm{~s}}$, A.I. Kononov ${ }^{47}$, R. Konoplich ${ }^{107, \mathrm{t}}$, N. Konstantinidis ${ }^{76}$, S. Koperny ${ }^{37}$, K. Korcyl ${ }^{38}$, K. Kordas ${ }^{153}$, A. Korn ${ }^{117}$, A. Korol ${ }^{106}$, I. Korolkov ${ }^{11}$, E.V. Korolkova ${ }^{138}$, V.A. Korotkov ${ }^{127}$, O. Kortner ${ }^{98}$, S. Kortner $^{98}$, V.V. Kostyukhin ${ }^{20}$, S. Kotov ${ }^{98}$, V.M. Kotov ${ }^{63}$, A. Kotwal ${ }^{44}$, C. Kourkoumelis ${ }^{8}$, V. Kouskoura ${ }^{153}$, A. Koutsman ${ }^{158 a}$, R. Kowalewski ${ }^{168}$, T.Z. Kowalski ${ }^{37}$, W. Kozanecki ${ }^{135}$, A.S. Kozhin ${ }^{127}$, V. Kral ${ }^{126}$, V.A. Kramarenko ${ }^{96}$, G. Kramberger ${ }^{73}$, M.W. Krasny ${ }^{77}$, A. Krasznahorkay ${ }^{107}$, J.K. Kraus ${ }^{20}$, S. Kreiss ${ }^{107}$, F. Krejci ${ }^{126}$, J. Kretzschmar ${ }^{72}$, N. Krieger ${ }^{53}$, P. Krieger ${ }^{157}$, K. Kroeninger ${ }^{53}$, H. Kroha ${ }^{98}$, J. Kroll ${ }^{119}$, J. Kroseberg ${ }^{20}$, J. Krstic ${ }^{12 a}$, U. Kruchonak ${ }^{63}$, H. Krüger ${ }^{20}$, T. Kruker ${ }^{16}$, N. Krumnack $^{62}$, Z.V. Krumshteyn ${ }^{63}$, T. Kubota ${ }^{85}$, S. Kuday ${ }^{3 a}$, S. Kuehn ${ }^{47}$, A. Kugel ${ }^{57 c}$, T. Kuhl ${ }^{41}$, D. Kuhn ${ }^{60}$, V. Kukhtin ${ }^{63}$, Y. Kulchitsky ${ }^{89}$, S. Kuleshov ${ }^{31 b}$, C. Kummer ${ }^{97}$, M. Kuna ${ }^{77}$, J. Kunkle ${ }^{119}$, A. Kupco ${ }^{124}$, H. Kurashige ${ }^{65}$, M. Kurata ${ }^{159}$, Y.A. Kurochkin ${ }^{89}$, V. Kus ${ }^{124}$, E.S. Kuwertz ${ }^{146}$, M. Kuze ${ }^{156}$, J. Kvita ${ }^{141}$, R. Kwee ${ }^{15}$, A. La Rosa ${ }^{48}$, L. La Rotonda ${ }^{36 a, 36 b}$, L. Labarga ${ }^{79}$, J. Labbe ${ }^{4}$, S. Lablak ${ }^{134 a}$, C. Lacasta ${ }^{166}$, F. Lacava ${ }^{131 a, 131 b}$, H. Lacker ${ }^{15}$, D. Lacour ${ }^{77}$, V.R. Lacuesta ${ }^{166}$, E. Ladygin ${ }^{63}$, R. Lafaye ${ }^{4}$, B. Laforge ${ }^{77}$, T. Lagouri ${ }^{79}$, S. Lai ${ }^{47}$, E. Laisne ${ }^{54}$, M. Lamanna ${ }^{29}$, L. Lambourne ${ }^{76}$, C.L. Lam-

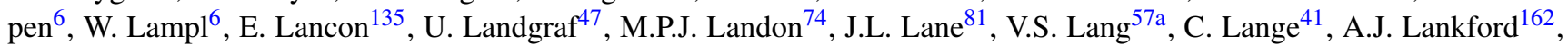
F. Lanni ${ }^{24}$, K. Lantzsch ${ }^{174}$, S. Laplace ${ }^{77}$, C. Lapoire ${ }^{20}$, J.F. Laporte ${ }^{135}$, T. Lari ${ }^{88 a}$, A. Larner ${ }^{117}$, M. Lassnig ${ }^{29}$, P. Laurelli $^{46}$, V. Lavorini ${ }^{36 a, 36 b}$, W. Lavrijsen ${ }^{14}$, P. Laycock ${ }^{72}$, O. Le Dortz ${ }^{77}$, E. Le Guirriec ${ }^{82}$, C. Le Maner ${ }^{157}$, E. Le Menedeu ${ }^{11}$, T. LeCompte ${ }^{5}$, F. Ledroit-Guillon ${ }^{54}$, H. Lee ${ }^{104}$, J.S.H. Lee ${ }^{115}$, S.C. Lee ${ }^{150}$, L. Lee ${ }^{175}$, M. Lefebvre ${ }^{168}$, M. Legendre ${ }^{135}$, F. Legger ${ }^{97}$, C. Leggett ${ }^{14}$, M. Lehmacher ${ }^{20}$, G. Lehmann Miotto ${ }^{29}$, X. Lei ${ }^{6}$, M.A.L. Leite ${ }^{23 d}$, R. Leitner ${ }^{125}$, D. Lellouch ${ }^{171}$, B. Lemmer ${ }^{53}$, V. Lendermann ${ }^{57 a}$, K.J.C. Leney ${ }^{144 b}$, T. Lenz ${ }^{104}$, G. Lenzen ${ }^{174}$, B. Lenzi ${ }^{29}$, K. Leonhardt ${ }^{43}$, S. Leontsinis $^{9}$, F. Lepold ${ }^{57 a}$, C. Leroy ${ }^{92}$, J-R. Lessard ${ }^{168}$, C.G. Lester $^{27}$, C.M. Lester ${ }^{119}$, J. Levêque ${ }^{4}$, D. Levin ${ }^{86}$, L.J. Levin-

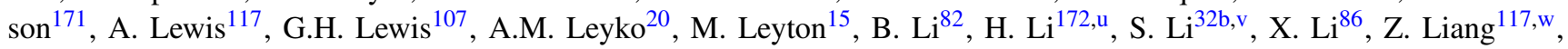

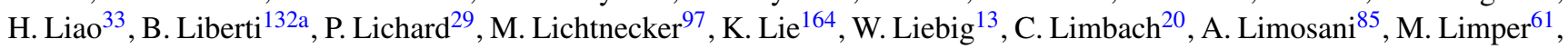
S.C. Lin $^{150, x}$, F. Linde ${ }^{104}$, J.T. Linnemann ${ }^{87}$, E. Lipeles ${ }^{119}$, A. Lipniacka ${ }^{13}$, T.M. Liss ${ }^{164}$, D. Lissauer ${ }^{24}$, A. Lister ${ }^{48}$,

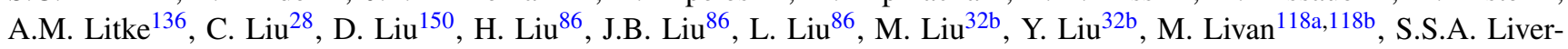
more $^{117}$, A. Lleres ${ }^{54}$, J. Llorente Merino ${ }^{79}$, S.L. Lloyd ${ }^{74}$, E. Lobodzinska ${ }^{41}$, P. Loch ${ }^{6}$, W.S. Lockman ${ }^{136}$, T. Loddenkoetter ${ }^{20}$, F.K. Loebinger ${ }^{81}$, A. Loginov ${ }^{175}$, C.W. Loh ${ }^{167}$, T. Lohse $^{15}$, K. Lohwasser ${ }^{47}$, M. Lokajicek ${ }^{124}$, V.P. Lombardo ${ }^{4}$, R.E. Long ${ }^{70}$, L. Lopes ${ }^{123 a}$, D. Lopez Mateos ${ }^{56}$, J. Lorenz ${ }^{97}$, N. Lorenzo Martinez ${ }^{114}$, M. Losada ${ }^{161}$, P. Loscutoff $^{14}$, F. Lo Sterzo ${ }^{131 a, 131 b}$, M.J. Losty ${ }^{158 a}$, X. Lou ${ }^{40}$, A. Lounis ${ }^{114}$, K.F. Loureiro ${ }^{161}$, J. Love ${ }^{21}$, P.A. Love ${ }^{70}$, A.J. Lowe ${ }^{142, e}$, F. Lu ${ }^{32 a}$, H.J. Lubatti $^{137}$, C. Luci ${ }^{131 a, 131 b}$, A. Lucotte ${ }^{54}$, A. Ludwig ${ }^{43}$, D. Ludwig ${ }^{41}$, I. Ludwig ${ }^{47}$, J. Ludwig ${ }^{47}$, F. Luehring ${ }^{59}$, G. Luijckx ${ }^{104}$, W. Lukas $^{60}$, D. Lumb ${ }^{47}$, L. Luminari131a, E. Lund ${ }^{116}$, B. Lund-Jensen ${ }^{146}$, B. Lundberg ${ }^{78}$, J. Lundberg ${ }^{145 a, 145 b}$, O. Lundberg ${ }^{145 a, 145 b}$, J. Lundquist ${ }^{35}$, M. Lungwitz ${ }^{80}$, D. Lynn ${ }^{24}$, E. Lytken ${ }^{78}$, H. Ma ${ }^{24}$, L.L. Ma ${ }^{172}$, G. Maccarrone ${ }^{46}$, A. Macchi$\mathrm{olo}^{98}$, B. Maček ${ }^{73}$, J. Machado Miguens ${ }^{123 a}$, R. Mackeprang ${ }^{35}$, R.J. Madaras ${ }^{14}$, H.J. Maddocks ${ }^{70}$, W.F. Mader ${ }^{43}$, R. Maenner $^{57 \mathrm{c}}$, T. Maeno ${ }^{24}$, P. Mättig ${ }^{174}$, S. Mättig ${ }^{41}$, L. Magnoni ${ }^{29}$, E. Magradze ${ }^{53}$, K. Mahboubi ${ }^{47}$, S. Mahmoud ${ }^{72}$, G. Mahout ${ }^{17}$, C. Maiani ${ }^{135}$, C. Maidantchik ${ }^{23 a}$, A. Maio ${ }^{123 a, b}$, S. Majewski ${ }^{24}$, Y. Makida ${ }^{64}$, N. Makovec ${ }^{114}$, P. Mal ${ }^{135}$, B. Malaescu ${ }^{29}$, Pa. Malecki ${ }^{38}$, P. Malecki ${ }^{38}$, V.P. Maleev ${ }^{120}$, F. Malek ${ }^{54}$, U. Mallik ${ }^{61}$, D. Malon ${ }^{5}$, C. Malone ${ }^{142}$, S. Maltezos ${ }^{9}$, V. Malyshev $^{106}$, S. Malyukov ${ }^{29}$, R. Mameghani ${ }^{97}$, J. Mamuzic ${ }^{12 b}$, A. Manabe ${ }^{64}$, L. Mandelli ${ }^{88 a}$, I. Mandić ${ }^{73}$, R. Mandrysch ${ }^{15}$, J. Maneira ${ }^{123 a}$, P.S. Mangeard ${ }^{87}$, L. Manhaes de Andrade Filho ${ }^{23 b}$, J.A. Manjarres Ramos ${ }^{135}$, A. Mann ${ }^{53}$, P.M. Manning ${ }^{136}$, A. Manousakis-Katsikakis ${ }^{8}$, B. Mansoulie ${ }^{135}$, A. Mapelli ${ }^{29}$, L. Mapelli ${ }^{29}$, L. March $^{79}$, J.F. Marchand ${ }^{28}$, F. Marchese ${ }^{132 a, 132 b}$, 
G. Marchiori $^{77}$, M. Marcisovsky ${ }^{124}$, C.P. Marino ${ }^{168}$, F. Marroquim ${ }^{23 a}$, Z. Marshall ${ }^{29}$, F.K. Martens ${ }^{157}$, L.F. Marti ${ }^{16}$, S. MartiGarcia $^{166}$, B. Martin ${ }^{29}$, B. Martin ${ }^{87}$, J.P. Martin${ }^{92}$, T.A. Martin ${ }^{17}$, V.J. Martin ${ }^{45}$, B. Martin dit Latour ${ }^{48}$, S. Martin-Haugh ${ }^{148}$, M. Martinez ${ }^{11}$, V. Martinez Outschoorn ${ }^{56}$, A.C. Martyniuk ${ }^{168}$, M. Marx ${ }^{81}$, F. Marzano ${ }^{131 a}$, A. Marzin ${ }^{110}$, L. Masetti ${ }^{80}$, T. Mashimo ${ }^{154}$, R. Mashinistov ${ }^{93}$, J. Masik ${ }^{81}$, A.L. Maslennikov ${ }^{106}$, I. Massa ${ }^{19 a, 19 b}$, G. Massaro ${ }^{104}$, N. Massol ${ }^{4}$, P. Mastrandrea $^{147}$, A. Mastroberardino ${ }^{36 a, 36 b}$, T. Masubuchi ${ }^{154}$, P. Matricon ${ }^{114}$, H. Matsunaga ${ }^{154}$, T. Matsushita ${ }^{65}$, C. Mattravers $^{117, c}$, J. Maurer ${ }^{82}$, S.J. Maxfield ${ }^{72}$, A. Mayne ${ }^{138}$, R. Mazini ${ }^{150}$, M. Mazur ${ }^{20}$, L. Mazzaferro ${ }^{132 a, 132 b}$, M. Mazzanti ${ }^{88 a}$, S.P. Mc Kee ${ }^{86}$, A. McCarn ${ }^{164}$, R.L. McCarthy ${ }^{147}$, T.G. McCarthy ${ }^{28}$, N.A. McCubbin ${ }^{128}$, K.W. McFarlane ${ }^{55,{ }^{*}}$, J.A. Mcfayden $^{138}$, G. Mchedlidze ${ }^{50 b}$, T. Mclaughlan ${ }^{17}$, S.J. McMahon ${ }^{128}$, R.A. McPherson ${ }^{168, k}$, A. Meade ${ }^{83}$, J. Mechnich ${ }^{104}$, M. Mechtel ${ }^{174}$, M. Medinnis ${ }^{41}$, R. Meera-Lebbai ${ }^{110}$, T. Meguro ${ }^{115}$, R. Mehdiyev ${ }^{92}$, S. Mehlhase ${ }^{35}$, A. Mehta ${ }^{72}$, K. Meier $^{57 a}$, B. Meirose ${ }^{78}$, C. Melachrinos ${ }^{30}$, B.R. Mellado Garcia ${ }^{172}$, F. Meloni ${ }^{88 a, 88 b}$, L. Mendoza Navas ${ }^{161}$, Z. Meng ${ }^{150, u}$, A. Mengarelli $^{19 a, 19 b}$, S. Menke ${ }^{98}$, E. Meoni ${ }^{160}$, K.M. Mercurio ${ }^{56}$, P. Mermod ${ }^{48}$, L. Merola ${ }^{101 a, 101 b}$, C. Meroni ${ }^{88 a}$, F.S. Merritt ${ }^{30}$, H. Merritt ${ }^{108}$, A. Messina ${ }^{29, y}$, J. Metcalfe ${ }^{102}$, A.S. Mete ${ }^{162}$, C. Meyer ${ }^{80}$, C. Meyer ${ }^{30}$, J-P. Meyer ${ }^{135}$, J. Meyer ${ }^{173}$, J. Meyer ${ }^{53}$, T.C. $M_{e y e r}{ }^{29}$, J. Miao ${ }^{32 d}$, S. Michal $^{29}$, L. Micu ${ }^{25 a}$, R.P. Middleton ${ }^{128}$, S. Migas ${ }^{72}$, L. Mijović ${ }^{135}$, G. Mikenberg ${ }^{171}$, M. Mikestikova ${ }^{124}$, M. Mikuž ${ }^{73}$, D.W. Miller ${ }^{30}$, R.J. Miller $^{87}$, W.J. Mills ${ }^{167}$, C. Mills ${ }^{56}$, A. Milov ${ }^{171}$, D.A. Milstead ${ }^{145 a, 145 b}$, D. Milstein ${ }^{171}$, A.A. Minaenko ${ }^{127}$, M. Miñano Moya ${ }^{166}$, I.A. Minashvili ${ }^{63}$, A.I. Mincer ${ }^{107}$, B. Mindur ${ }^{37}$, M. Mineev ${ }^{63}$, Y. Ming ${ }^{172}$, L.M. Mir $^{11}$, G. Mirabelli ${ }^{131 a}$, J. Mitrevski ${ }^{136}$, V.A. Mitsou ${ }^{166}$, S. Mitsui ${ }^{64}$, P.S. Miyagawa ${ }^{138}$, J.U. Mjörnmark $^{78}$, T. Moa ${ }^{145 a, 145 b}$, V. Moeller ${ }^{27}$, K. Mönig ${ }^{41}$, N. Möser ${ }^{20}$, S. Mohapatra ${ }^{147}$, W. Mohr ${ }^{47}$, R. Moles-Valls ${ }^{166}$, J. Monk ${ }^{76}$, E. Monnier ${ }^{82}$, J. Montejo Berlingen ${ }^{11}$, F. Monticelli ${ }^{69}$, S. Monzani ${ }^{19 a, 19 b}$, R.W. Moore ${ }^{2}$, G.F. Moorhead ${ }^{85}$, C. Mora Herrera $^{48}$, A. Moraes ${ }^{52}$, N. Morange ${ }^{135}$, J. Morel ${ }^{53}$, G. Morello ${ }^{36 a, 36 b}$, D. Moreno ${ }^{80}$, M. Moreno Llácer ${ }^{166}$, P. Morettini ${ }^{49 a}$, M. Morgenstern ${ }^{43}$, M. Morii ${ }^{56}$, A.K. Morley ${ }^{29}$, G. Mornacchi ${ }^{29}$, J.D. Morris ${ }^{74}$, L. Morvaj ${ }^{100}$, H.G. Moser ${ }^{98}$, M. Mosidze ${ }^{50 b}$, J. Moss ${ }^{108}$, R. Mount ${ }^{142}$, E. Mountricha ${ }^{9, z}$, S.V. Mouraviev ${ }^{93, *}$, E.J.W. Moyse ${ }^{83}$, F. Mueller ${ }^{57 a}$, J. Mueller ${ }^{122}$, K. Mueller ${ }^{20}$, T.A. Müller ${ }^{97}$, T. Mueller ${ }^{80}$, D. Muenstermann ${ }^{29}$, Y. Munwes ${ }^{152}$, W.J. Murray ${ }^{128}$, I. Mussche ${ }^{104}$, E. Musto ${ }^{101 a, 101 b}$, A.G. Myagkov ${ }^{127}$, M. Myska ${ }^{124}$, J. Nadal ${ }^{11}$, K. Nagai ${ }^{159}$, R. Nagai ${ }^{156}$, K. Nagano ${ }^{64}$, A. Nagarkar ${ }^{108}$, Y. Nagasaka ${ }^{58}$, M. Nagel ${ }^{98}$, A.M. Nairz ${ }^{29}$, Y. Nakahama ${ }^{29}$, K. Nakamura ${ }^{154}$, T. Nakamura ${ }^{154}$, I. Nakano ${ }^{109}$, G. Nanava ${ }^{20}$, A. Napier ${ }^{160}$, R. Narayan ${ }^{57 b}$, M. Nash ${ }^{76, c}$, T. Nattermann ${ }^{20}$, T. Naumann ${ }^{41}$, G. Navarro ${ }^{161}$, H.A. Neal ${ }^{86}$, P.Yu. Nechaeva ${ }^{93}$, T.J. Neep ${ }^{81}$, A. Negri ${ }^{118 a, 118 b}$, G. Negrii ${ }^{29}$, M. Negrini ${ }^{19 a}$, S. Nektarijevic ${ }^{48}$, A. Nelson ${ }^{162}$, T.K. Nelson ${ }^{142}$, S. Nemecek ${ }^{124}$, P. Nemethy ${ }^{107}$, A.A. Nepomuceno ${ }^{23 a}$, M. Nessi ${ }^{29, a a}$, M.S. Neubauer ${ }^{164}$, A. Neusiedl ${ }^{80}$, R.M. Neves ${ }^{107}$, P. Nevski ${ }^{24}$, P.R. Newman ${ }^{17}$, V. Nguyen Thi Hong ${ }^{135}$, R.B. Nickerson ${ }^{117}$, R. Nicolaidou ${ }^{135}$, B. Nicquevert ${ }^{29}$, F. Niedercorn ${ }^{114}$, J. Nielsen ${ }^{136}$, N. Nikiforou $^{34}$, A. Nikiforov ${ }^{15}$, V. Nikolaenko ${ }^{127}$, I. Nikolic-Audit ${ }^{77}$, K. Nikolics ${ }^{48}$, K. Nikolopoulos ${ }^{17}$, H. Nilsen ${ }^{47}$, P. Nilsson ${ }^{7}$, Y. Ninomiya ${ }^{154}$, A. Nisati ${ }^{131 a}$, R. Nisius ${ }^{98}$, T. Nobe ${ }^{156}$, L. Nodulman ${ }^{5}$, M. Nomachi ${ }^{115}$, I. Nomidis ${ }^{153}$, S. Norberg ${ }^{110}$, M. Nordberg ${ }^{29}$, P.R. Norton ${ }^{128}$, J. Novakova ${ }^{125}$, M. Nozaki ${ }^{64}$, L. Nozka ${ }^{112}$, I.M. Nugent ${ }^{158 a}$, A.-E. Nuncio-Quiroz ${ }^{20}$, G. Nunes Hanninger ${ }^{85}$, T. Nunnemann ${ }^{97}$, E. Nurse ${ }^{76}$, B.J. O’Brien ${ }^{45}$, S.W. O’Neale ${ }^{17, *}$, D.C. O’Neil ${ }^{141}$, V. O’Shea ${ }^{52}$, L.B. Oakes ${ }^{97}$, F.G. Oakham ${ }^{28, \mathrm{~d}}$, H. Oberlack ${ }^{98}$, J. Ocariz ${ }^{77}$, A. Ochi ${ }^{65}$, S. Oda ${ }^{68}$, S. Odaka ${ }^{64}$, J. Odier ${ }^{82}$, H. Ogren $^{59}$, A. Oh $^{81}$, S.H. $\mathrm{Oh}^{44}$, C.C. $\mathrm{Ohm}^{29}$, T. Ohshima ${ }^{100}$, H. Okawa ${ }^{24}$, Y. Okumura ${ }^{30}$, T. Okuyama ${ }^{154}$, A. Olariu ${ }^{25 a}$, A.G. Olchevski ${ }^{63}$, S.A. Olivares Pino ${ }^{31 a}$, M. Oliveira ${ }^{123 a, h}$, D. Oliveira Damazio ${ }^{24}$, E. Oliver Garcia ${ }^{166}$, D. Olivito ${ }^{119}$, A. Olszewski ${ }^{38}$, J. Ol- $^{-}$ szowska $^{38}$, A. Onofre ${ }^{123 a, a b}$, P.U.E. Onyisi ${ }^{30}$, C.J. Oram ${ }^{158 a}$, M.J. Oreglia ${ }^{30}$, Y. Oren ${ }^{152}$, D. Orestano ${ }^{133 a, 133 b}$, N. Orlando $^{71 \mathrm{a}, 71 \mathrm{~b}}$, I. Orlov ${ }^{106}$, C. Oropeza Barrera ${ }^{52}$, R.S. Orr ${ }^{157}$, B. Osculati ${ }^{49 a, 49 b}$, R. Ospanov ${ }^{119}$, C. Osuna ${ }^{11}$, G. Otero y Garzon $^{26}$, J.P. Ottersbach ${ }^{104}$, M. Ouchrif ${ }^{134 d}$, E.A. Ouellette ${ }^{168}$, F. Ould-Saada ${ }^{116}$, A. Ouraou ${ }^{135}$, Q. Ouyang ${ }^{32 a}$, A. Ovcharova ${ }^{14}$, M. Owen ${ }^{81}$, S. Owen ${ }^{138}$, V.E. Ozcan ${ }^{18 a}$, N. Ozturk ${ }^{7}$, A. Pacheco Pages ${ }^{11}$, C. Padilla Aranda ${ }^{11}$, S. Pagan Griso ${ }^{14}$, E. Paganis $^{138}$, C. Pahl ${ }^{98}$, F. Paige ${ }^{24}$, P. Pais ${ }^{83}$, K. Pajchel ${ }^{116}$, G. Palacino ${ }^{158 b}$, C.P. Paleari ${ }^{6}$, S. Palestini ${ }^{29}$, D. Pallin ${ }^{33}$, A. Palma ${ }^{123 a}$, J.D. Palmer ${ }^{17}$, Y.B. Pan ${ }^{172}$, E. Panagiotopoulou ${ }^{9}$, P. Pani ${ }^{104}$, N. Panikashvili ${ }^{86}$, S. Panitkin ${ }^{24}$, D. Pantea ${ }^{25 a}$, A. Papadelis ${ }^{145 a}$, Th.D. Papadopoulou ${ }^{9}$, A. Paramonov 5 , D. Paredes Hernandez ${ }^{33}$, W. Park ${ }^{24, a c}$, M.A. Parker ${ }^{27}$, F. Parodi ${ }^{49 a, 49 b}$, J.A. Parsons $^{34}$, U. Parzefall ${ }^{47}$, S. Pashapour ${ }^{53}$, E. Pasqualucci ${ }^{131 a}$, S. Passaggio ${ }^{49 a}$, A. Passeri ${ }^{133 a}$, F. Pastore ${ }^{133 a, 133 b, *}$, Fr. Pastore $^{75}$, G. Pásztor ${ }^{48, a d}$, S. Pataraia ${ }^{174}$, N. Patel ${ }^{149}$, J.R. Pater ${ }^{81}$, S. Patricelli ${ }^{101 a, 101 b}$, T. Pauly ${ }^{29}$, M. Pecsy ${ }^{143 a}$, M.I. Pedraza Morales $^{172}$, S.V. Peleganchuk ${ }^{106}$, D. Pelikan ${ }^{165}$, H. Peng ${ }^{32 b}$, B. Penning ${ }^{30}$, A. Penson ${ }^{34}$, J. Penwell ${ }^{59}$, M. Perantoni ${ }^{23 a}$, K. Perez ${ }^{34, a e}$, T. Perez Cavalcanti ${ }^{41}$, E. Perez Codina ${ }^{158 a}$, M.T. Pérez García-Estañ ${ }^{166}$, V. Perez Reale ${ }^{34}$, L. Perini ${ }^{88 a, 88 b}$, H. Pernegger ${ }^{29}$, R. Perrino ${ }^{71 a}$, P. Perrodo ${ }^{4}$, V.D. Peshekhonov ${ }^{63}$, K. Peters ${ }^{29}$, B.A. Petersen ${ }^{29}$, J. Petersen ${ }^{29}$, T.C. Pe$\operatorname{tersen}^{35}$, E. Petit ${ }^{4}$, A. Petridis ${ }^{153}$, C. Petridou ${ }^{153}$, E. Petrolo ${ }^{131 a}$, F. Petrucci ${ }^{133 a, 133 b}$, D. Petschull ${ }^{41}$, M. Petteni ${ }^{141}$, R. Pezoa $^{31 b}$, A. Phan ${ }^{85}$, P.W. Phillips ${ }^{128}$, G. Piacquadio ${ }^{29}$, A. Picazio ${ }^{48}$, E. Piccaro ${ }^{74}$, M. Piccinini ${ }^{19 a, 19 b}$, S.M. Piec ${ }^{41}$, R. Piegaia $^{26}$, D.T. Pignotti ${ }^{108}$, J.E. Pilcher ${ }^{30}$, A.D. Pilkington ${ }^{81}$, J. Pina ${ }^{123 a, b}$, M. Pinamonti ${ }^{163 a, 163 c}$, A. Pinder ${ }^{117}$, J.L. Pinfold ${ }^{2}$, B. Pinto ${ }^{123 a}$, C. Pizio ${ }^{88 a, 88 b}$, M. Plamondon ${ }^{168}$, M.-A. Pleier ${ }^{24}$, E. Plotnikova ${ }^{63}$, A. Poblaguev ${ }^{24}$, S. Poddar ${ }^{57 a}$, F. Podlyski ${ }^{33}$, L. Poggioli ${ }^{114}$, M. Pohl $^{48}$, G. Polesello ${ }^{118 a}$, A. Policicchio ${ }^{36 a, 36 b}$, A. Polini ${ }^{19 a}$, J. Poll ${ }^{74}$, V. Polychronakos ${ }^{24}$, D. Pomeroy ${ }^{22}$, K. Pommès ${ }^{29}$, L. Pontecorvo ${ }^{131 a}$, B.G. Pope ${ }^{87}$, G.A. Popeneciu ${ }^{25 a}$, D.S. Popovic ${ }^{12 a}$, A. Poppleton ${ }^{29}$, X. Portell Bueso ${ }^{29}$, 
G.E. Pospelov ${ }^{98}$, S. Pospisil ${ }^{126}$, I.N. Potrap ${ }^{98}$, C.J. Potter ${ }^{148}$, C.T. Potter ${ }^{113}$, G. Poulard ${ }^{29}$, J. Poveda ${ }^{59}$, V. Pozdnyakov ${ }^{63}$,

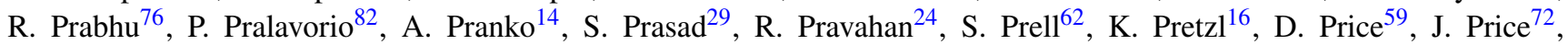
L.E. Price ${ }^{5}$, D. Prieur ${ }^{122}$, M. Primavera ${ }^{71 a}$, K. Prokofiev ${ }^{107}$, F. Prokoshin ${ }^{31 b}$, S. Protopopescu ${ }^{24}$, J. Proudfoot ${ }^{5}$, X. Prudent ${ }^{43}$, M. Przybycien ${ }^{37}$, H. Przysiezniak ${ }^{4}$, S. Psoroulas ${ }^{20}$, E. Ptacek ${ }^{113}$, E. Pueschel ${ }^{83}$, J. Purdham ${ }^{86}$, M. Purohit ${ }^{24, a c}$, P. Puzo $^{114}$, Y. Pylypchenko ${ }^{61}$, J. Qian ${ }^{86}$, A. Quadt ${ }^{53}$, D.R. Quarrie ${ }^{14}$, W.B. Quayle ${ }^{172}$, F. Quinonez ${ }^{31 a}$, M. Raas ${ }^{103}$, V. Radescu ${ }^{41}$, P. Radloff ${ }^{113}$, T. Rador ${ }^{18 a}$, F. Ragusa ${ }^{88 a}$,88b, G. Rahal ${ }^{177}$, A.M. Rahimi ${ }^{108}$, D. Rahm ${ }^{24}$, S. Rajagopalan ${ }^{24}$, M. Rammensee ${ }^{47}$, M. Rammes ${ }^{140}$, A.S. Randle-Conde ${ }^{39}$, K. Randrianarivony ${ }^{28}$, F. Rauscher ${ }^{97}$, T.C. Rave ${ }^{47}$, M. Raymond ${ }^{29}$, A.L. Read ${ }^{116}$, D.M. Rebuzzi ${ }^{118 a, 118 b}$, A. Redelbach ${ }^{173}$, G. Redlinger ${ }^{24}$, R. Reece ${ }^{119}$, K. Reeves ${ }^{40}$, E. Reinherz-Aronis ${ }^{152}$, A. Reinsch ${ }^{113}$, I. Reisinger ${ }^{42}$, C. Rembser $^{29}$, Z.L. Ren ${ }^{150}$, A. Renaud ${ }^{114}$, M. Rescigno ${ }^{131 a}$, S. Resconi ${ }^{88 a}$, B. Resende ${ }^{135}$, P. Reznicek ${ }^{97}$, R. Rezvani ${ }^{157}$, R. Richter ${ }^{98}$, E. Richter-Was ${ }^{4, a f}$, M. Ridel ${ }^{77}$, M. Rijpstra ${ }^{104}$, M. Rijssenbeek ${ }^{147}$, A. Rimoldi1 ${ }^{18 a, 118 b}$, L. Rinaldi ${ }^{19 a}$, R.R. Rios ${ }^{39}$, I. Riu ${ }^{11}$, G. Rivoltella ${ }^{88 a, 88 b}$, F. Rizatdinova ${ }^{111}$, E. Rizvi ${ }^{74}$, S.H. Robertson ${ }^{84, k}$, A. RobichaudVeronneau $^{117}$, D. Robinson ${ }^{27}$, J.E.M. Robinson ${ }^{81}$, A. Robson ${ }^{52}$, J.G. Rocha de Lima ${ }^{105}$, C. Roda ${ }^{121 a, 121 b}$, D. Roda Dos San$\operatorname{tos}^{29}$, A. Roe ${ }^{53}$, S. Roe ${ }^{29}$, O. Røhne ${ }^{116}$, S. Rolli ${ }^{160}$, A. Romaniouk ${ }^{95}$, M. Romano ${ }^{19 a, 19 b}$, G. Romeo ${ }^{26}$, E. Romero Adam $^{166}$, L. Roos ${ }^{77}$, E. Ros ${ }^{166}$, S. Rosati ${ }^{131 a}$, K. Rosbach ${ }^{48}$, A. Rose ${ }^{148}$, M. Rose ${ }^{75}$, G.A. Rosenbaum ${ }^{157}$, E.I. Rosenberg $^{62}$, P.L. Rosendahl ${ }^{13}$, O. Rosenthal ${ }^{140}$, L. Rosselet ${ }^{48}$, V. Rossetti ${ }^{11}$, E. Rossi ${ }^{131 a, 131 b}$, L.P. Rossi ${ }^{49 a}$, M. Rotaru ${ }^{25 a}$, I. Roth ${ }^{171}$, J. Rothberg ${ }^{137}$, D. Rousseau ${ }^{114}$, C.R. Royon ${ }^{135}$, A. Rozanov ${ }^{82}$, Y. Rozen ${ }^{151}$, X. Ruan ${ }^{32 a, a g}$, F. Rubbo ${ }^{11}$, I. Rubinskiy $^{41}$, B. Ruckert ${ }^{97}$, N. Ruckstuhl ${ }^{104}$, V.I. Rud ${ }^{96}$, C. Rudolph ${ }^{43}$, G. Rudolph ${ }^{60}$, F. Rühr ${ }^{6}$, A. Ruiz-Martinez ${ }^{62}$, L. Rumyant$\operatorname{sev}^{63}$, Z. Rurikova ${ }^{47}$, N.A. Rusakovich ${ }^{63}$, J.P. Rutherfoord ${ }^{6}$, C. Ruwiedel ${ }^{14, *}$, P. Ruzicka ${ }^{124}$, Y.F. Ryabov ${ }^{120}$, P. Ryan ${ }^{87}$, M. Rybar ${ }^{125}$, G. Rybkin ${ }^{114}$, N.C. Ryder ${ }^{117}$, A.F. Saavedra ${ }^{149}$, I. Sadeh ${ }^{152}$, H.F-W. Sadrozinski ${ }^{136}$, R. Sadykov ${ }^{63}$, F. Safai Tehrani $^{131 a}$, H. Sakamoto ${ }^{154}$, G. Salamanna ${ }^{74}$, A. Salamon ${ }^{132 a}$, M. Saleem ${ }^{110}$, D. Salek ${ }^{29}$, D. Salihagic ${ }^{98}$, A. Salnikov ${ }^{142}$, J. Salt ${ }^{166}$, B.M. Salvachua Ferrando ${ }^{5}$, D. Salvatore ${ }^{36 a, 36 b}$, F. Salvatore ${ }^{148}$, A. Salvucci ${ }^{103}$, A. Salzburger ${ }^{29}$, D. Sampsonidis $^{153}$, B.H. Samset ${ }^{116}$, A. Sanchez ${ }^{101 a, 101 b}$, V. Sanchez Martinez ${ }^{166}$, H. Sandaker ${ }^{13}$, H.G. Sander ${ }^{80}$, M.P. Sanders ${ }^{97}$, M. Sandhoff ${ }^{174}$, T. Sandoval ${ }^{27}$, C. Sandoval ${ }^{161}$, R. Sandstroem ${ }^{98}$, D.P.C. Sankey ${ }^{128}$, A. Sansoni ${ }^{46}$, C. Santamarina Rios ${ }^{84}$, C. Santoni ${ }^{33}$, R. Santonico ${ }^{132 a, 132 b}$, H. Santos ${ }^{123 a}$, J.G. Saraiva ${ }^{123 a}$, T. Sarangi ${ }^{172}$, E. Sarkisyan-Grinbaum ${ }^{7}$, F. Sarri ${ }^{121 a, 121 b}$, G. Sartisohn ${ }^{174}$, O. Sasaki ${ }^{64}$, Y. Sasaki ${ }^{154}$, N. Sasao ${ }^{66}$, I. Satsounkevitch ${ }^{89}$, G. Sauvage, ${ }^{4 *}$, E. Sauvan ${ }^{4}$, J.B. Sauvan ${ }^{114}$, P. Savard ${ }^{157, d}$, V. Savinov ${ }^{122}$, D.O. Savu ${ }^{29}$, L. Sawyer ${ }^{24, m}$, D.H. Saxon ${ }^{52}$, J. Saxon ${ }^{119}$, C. Sbarra ${ }^{19 a}$, A. Sbrizzi ${ }^{19 a, 19 b}$, D.A. Scannicchio ${ }^{162}$, M. Scarcella ${ }^{149}$, J. Schaarschmidt ${ }^{114}$, P. Schacht ${ }^{98}$, D. Schaefer ${ }^{119}$, U. Schäfer ${ }^{80}$, S. Schaepe ${ }^{20}$, S. Schaetzel ${ }^{57 b}$, A.C. Schaffer ${ }^{114}$, D. Schaile ${ }^{97}$, R.D. Schamberger ${ }^{147}$, A.G. Schamov ${ }^{106}$, V. Scharf ${ }^{57 a}$, V.A. Schegelsky $^{120}$, D. Scheirich ${ }^{86}$, M. Schernau ${ }^{162}$, M.I. Scherzer ${ }^{34}$, C. Schiavi ${ }^{49 a, 49 b}$, J. Schieck ${ }^{97}$, M. Schioppa ${ }^{36 a, 36 b}$, S. Schlenker ${ }^{29}$, E. Schmidt ${ }^{47}$, K. Schmieden ${ }^{20}$, C. Schmitt ${ }^{80}$, S. Schmitt ${ }^{57 b}$, M. Schmitz ${ }^{20}$, B. Schneider ${ }^{16}$, U. Schnoor ${ }^{43}$, A. Schoening ${ }^{57 b}$, A.L.S. Schorlemmer ${ }^{53}$, M. Schott ${ }^{29}$, D. Schouten ${ }^{158 a}$, J. Schovancova ${ }^{124}$, M. Schram ${ }^{84}$, C. Schroeder ${ }^{80}$, N. Schroer ${ }^{57 c}$, M.J. Schultens ${ }^{20}$, J. Schultes ${ }^{174}$, H.-C. Schultz-Coulon ${ }^{57 a}$, H. Schulz ${ }^{15}$, M. Schumacher ${ }^{47}$, B.A. Schumm ${ }^{136}$, Ph. Schune $^{135}$, C. Schwanenberger ${ }^{81}$, A. Schwartzman ${ }^{142}$, Ph. Schwemling ${ }^{77}$, R. Schwienhorst ${ }^{87}$, R. Schwierz ${ }^{43}$, J. Schwindling ${ }^{135}$, T. Schwindt ${ }^{20}$, M. Schwoerer ${ }^{4}$, G. Sciolla ${ }^{22}$, W.G. Scott ${ }^{128}$, J. Searcy ${ }^{113}$, G. Sedov ${ }^{41}$, E. Sedykh ${ }^{120}$, S.C. Seidel ${ }^{102}$, A. Sei$\operatorname{den}^{136}$, F. Seifert ${ }^{43}$, J.M. Seixas ${ }^{23 a}$, G. Sekhniaidze ${ }^{101 a}$, S.J. Sekula ${ }^{39}$, K.E. Selbach ${ }^{45}$, D.M. Seliverstov ${ }^{120}$, B. Sellden ${ }^{145 a}$, G. Sellers ${ }^{72}$, M. Seman ${ }^{143 b}$, N. Semprini-Cesari ${ }^{19 a, 19 b}$, C. Serfon ${ }^{97}$, L. Serin ${ }^{114}$, L. Serkin ${ }^{53}$, R. Seuster ${ }^{98}$, H. Severini ${ }^{110}$, A. Sfyrla ${ }^{29}$, E. Shabalina ${ }^{53}$, M. Shamim ${ }^{113}$, L.Y. Shan ${ }^{32 a}$, J.T. Shank ${ }^{21}$, Q.T. Shao ${ }^{85}$, M. Shapiro ${ }^{14}$, P.B. Shatalov ${ }^{94}$, K. Shaw ${ }^{163 a, 163 c}$, D. Sherman ${ }^{175}$, P. Sherwood ${ }^{76}$, A. Shibata ${ }^{107}$, S. Shimizu ${ }^{29}$, M. Shimojima ${ }^{99}$, T. Shin ${ }^{55}$, M. Shiyakova ${ }^{63}$, A. Shmeleva ${ }^{93}$, M.J. Shochet ${ }^{30}$, D. Short ${ }^{117}$, S. Shrestha ${ }^{62}$, E. Shulga ${ }^{95}$, M.A. Shupe ${ }^{6}$, P. Sicho ${ }^{124}$, A. Sidoti ${ }^{131 a}$, F. Siegert ${ }^{47}$, Dj. Sijacki ${ }^{12 a}$, O. Silbert ${ }^{171}$, J. Silva ${ }^{123 a}$, Y. Silver ${ }^{152}$, D. Silverstein ${ }^{142}$, S.B. Silverstein ${ }^{145 a}$, V. Simak ${ }^{126}$, O. Simard ${ }^{135}$, Lj. Simic ${ }^{12 a}$, S. Simion ${ }^{114}$, E. Simioni ${ }^{80}$, B. Simmons ${ }^{76}$, R. Simoniello ${ }^{88 a, 88 b}$, M. Simonyan ${ }^{35}$, P. Sinervo ${ }^{157}$, N.B. Sinev ${ }^{113}$, V. Sipica ${ }^{140}$, G. Siragusa ${ }^{173}$, A. Sircar ${ }^{24}$, A.N. Sisakyan ${ }^{63,{ }^{*}}$, S.Yu. Sivoklokov ${ }^{96}$, J. Sjölin ${ }^{145 a, 145 b}$, T.B. Sjursen ${ }^{13}$, L.A. Skinnari $^{14}$, H.P. Skottowe ${ }^{56}$, K. Skovpen ${ }^{106}$, P. Skubic ${ }^{110}$, M. Slater ${ }^{17}$, T. Slavicek ${ }^{126}$, K. Sliwa ${ }^{160}$, V. Smakhtin ${ }^{171}$, B.H. Smart ${ }^{45}$, S.Yu. Smirnov ${ }^{95}$, Y. Smirnov ${ }^{95}$, L.N. Smirnova ${ }^{96}$, O. Smirnova $^{78}$, B.C. Smith $^{56}$, D. Smith ${ }^{142}$, K.M. Smith ${ }^{52}$, M. Smizanska $^{70}$, K. Smolek ${ }^{126}$, A.A. Snesarev ${ }^{93}$, S.W. Snow ${ }^{81}$, J. Snow ${ }^{110}$, S. Snyder ${ }^{24}$, R. Sobie ${ }^{168, k}$, J. Sodomka ${ }^{126}$, A. Soffer ${ }^{152}$, C.A. Solans ${ }^{166}$, M. Solar ${ }^{126}$, J. Solc ${ }^{126}$, E.Yu. Soldatov ${ }^{95}$, U. Soldevila ${ }^{166}$, E. Solfaroli Camillocci131a,131b , A.A. Solodkov $^{127}$, O.V. Solovyanov ${ }^{127}$, V. Solovyev ${ }^{120}$, N. Soni ${ }^{85}$, V. Sopko ${ }^{126}$, B. Sopko ${ }^{126}$, M. Sosebee ${ }^{7}$, R. Soualah ${ }^{163 a, 163 c}$, A. Soukharev ${ }^{106}$, S. Spagnolo ${ }^{71 a, 71 b}$, F. Spanò ${ }^{75}$, R. Spighi ${ }^{19 a}$, G. Spigo ${ }^{29}$, R. Spiwoks ${ }^{29}$, M. Spousta ${ }^{125, \text { ah }}$, T. Spreitzer ${ }^{157}$, B. Spurlock ${ }^{7}$, R.D. St. Denis ${ }^{52}$, J. Stahlman ${ }^{119}$, R. Stamen ${ }^{57 a}$, E. Stanecka ${ }^{38}$, R.W. Stanek ${ }^{5}$, C. Stanescu ${ }^{133 a}$, M. StanescuBellu $^{41}$, S. Stapnes ${ }^{116}$, E.A. Starchenko ${ }^{127}$, J. Stark ${ }^{54}$, P. Staroba ${ }^{124}$, P. Starovoitov ${ }^{41}$, R. Staszewski ${ }^{38}$, A. Staude ${ }^{97}$, P. Stavina ${ }^{143 a}$,* G. Steele ${ }^{52}$, P. Steinbach ${ }^{43}$, P. Steinberg ${ }^{24}$, I. Stekl ${ }^{126}$, B. Stelzer ${ }^{141}$, H.J. Stelzer ${ }^{87}$, O. Stelzer-Chilton ${ }^{158 a}$, H. Stenzel ${ }^{51}$, S. Stern ${ }^{98}$, G.A. Stewart ${ }^{29}$, J.A. Stillings ${ }^{20}$, M.C. Stockton ${ }^{84}$, K. Stoerig ${ }^{47}$, G. Stoicea ${ }^{25 a}$, S. Stonjek ${ }^{98}$, P. Strachota ${ }^{125}$, A.R. Stradling ${ }^{7}$, A. Straessner ${ }^{43}$, J. Strandberg ${ }^{146}$, S. Strandberg ${ }^{145 a, 145 b}$, A. Strandlie ${ }^{116}$, M. Strang ${ }^{108}$, 
E. Strauss ${ }^{142}$, M. Strauss ${ }^{110}$, P. Strizenec ${ }^{143 b}$, R. Ströhmer ${ }^{173}$, D.M. Strom ${ }^{113}$, J.A. Strong ${ }^{75, *}$, R. Stroynowski ${ }^{39}$, J. Strube ${ }^{128}$, B. Stugu ${ }^{13}$, I. Stumer ${ }^{24, *}$, J. Stupak ${ }^{147}$, P. Sturm ${ }^{174}$, N.A. Styles ${ }^{41}$, D.A. Soh ${ }^{150, w}$, D. Su ${ }^{142}$, HS. Subramania ${ }^{2}$, A. Succurro $^{11}$, Y. Sugaya ${ }^{115}$, C. Suhr ${ }^{105}$, M. Suk ${ }^{125}$, V.V. Sulin ${ }^{93}$, S. Sultansoy ${ }^{3 d}$, T. Sumida ${ }^{66}$, X. Sun ${ }^{54}$, J.E. Sundermann ${ }^{47}$, K. Suruliz ${ }^{138}$, G. Susinno ${ }^{36 a, 36 b}$, M.R. Sutton ${ }^{148}$, Y. Suzuki ${ }^{64}$, Y. Suzuki ${ }^{65}$, M. Svatos ${ }^{124}$, S. Swedish ${ }^{167}$, I. Sykora ${ }^{143 a}$, T. Sykora ${ }^{125}$, J. Sánchez ${ }^{166}$, D. Ta ${ }^{104}$, K. Tackmann ${ }^{41}$, A. Taffard ${ }^{162}$, R. Tafirout ${ }^{158 a}$, N. Taiblum ${ }^{152}$, Y. Takahashi ${ }^{100}$, H. Takai ${ }^{24}$, R. Takashima ${ }^{67}$, H. Takeda ${ }^{65}$, T. Takeshita ${ }^{139}$, Y. Takubo ${ }^{64}$, M. Talby ${ }^{82}$, A. Talyshev ${ }^{106, f}$, M.C. Tamsett ${ }^{24}$, J. Tanaka ${ }^{154}$, R. Tanaka ${ }^{114}$, S. Tanaka ${ }^{130}$, S. Tanaka ${ }^{64}$, A.J. Tanasijczuk ${ }^{141}$, K. Tani ${ }^{65}$, N. Tannoury ${ }^{82}$, S. Tapprogge ${ }^{80}$, D. $\operatorname{Tardif}^{157}$, S. Tarem ${ }^{151}$, F. Tarrade ${ }^{28}$, G.F. Tartarelli ${ }^{88 a}$, P. Tas ${ }^{125}$, M. Tasevsky ${ }^{124}$, E. Tassi ${ }^{36 a, 36 b}$, M. Tatarkhanov ${ }^{14}$, Y. Tayalati ${ }^{134 \mathrm{~d}}$, C. Taylor $^{76}$, F.E. Taylor ${ }^{91}$, G.N. Taylor ${ }^{85}$, W. Taylor ${ }^{158 b}$, M. Teinturier ${ }^{114}$, M. Teixeira Dias Castanheira ${ }^{74}$, P. Teixeira-Dias ${ }^{75}$, K.K. Temming ${ }^{47}$, H. Ten Kate ${ }^{29}$, P.K. Teng ${ }^{150}$, S. Terada ${ }^{64}$, K. Terashi ${ }^{154}$, J. Terron ${ }^{79}$, M. Testa ${ }^{46}$, R.J. Teuscher ${ }^{157, k}$, J. Therhaag ${ }^{20}$, T. Theveneaux-Pelzer ${ }^{77}$, S. Thoma ${ }^{47}$, J.P. Thomas ${ }^{17}$, E.N. Thompson ${ }^{34}$, P.D. Thomp$\operatorname{son}^{17}$, P.D. Thompson ${ }^{157}$, A.S. Thompson ${ }^{52}$, L.A. Thomsen ${ }^{35}$, E. Thomson ${ }^{119}$, M. Thomson ${ }^{27}$, W.M. Thong ${ }^{85}$, R.P. Thun ${ }^{86}$, F. Tian $^{34}$, M.J. Tibbetts ${ }^{14}$, T. Tic ${ }^{124}$, V.O. Tikhomirov ${ }^{93}$, Y.A. Tikhonov ${ }^{106, f}$, S. Timoshenko ${ }^{95}$, P. Tipton ${ }^{175}$, S. Tisserant $^{82}$, T. Todorov ${ }^{4}$, S. Todorova-Nova ${ }^{160}$, B. Toggerson ${ }^{162}$, J. Tojo ${ }^{68}$, S. Tokár ${ }^{143 a}$, K. Tokushuku ${ }^{64}$, K. Tollefson ${ }^{87}$, M. Tomoto $^{100}$, L. Tompkins ${ }^{30}$, K. Toms ${ }^{102}$, A. Tonoyan ${ }^{13}$, C. Topfel ${ }^{16}$, N.D. Topilin ${ }^{63}$, I. Torchiani2 ${ }^{29}$, E. Torrence ${ }^{113}$, H. Torres ${ }^{77}$, E. Torró Pastor ${ }^{166}$, J. Toth ${ }^{82, \text { ad }}$, F. Touchard ${ }^{82}$, D.R. Tovey ${ }^{138}$, T. Trefzger ${ }^{173}$, L. Tremblet $^{29}$, A. Tricoli ${ }^{29}$, I.M. Trigger ${ }^{158 a}$, S. Trincaz-Duvoid ${ }^{77}$, M.F. Tripiana ${ }^{69}$, N. Triplett ${ }^{24}$, W. Trischuk ${ }^{157}$, B. Trocmé ${ }^{54}$, C. Troncon ${ }^{88 a}$, M. Trottier-McDonald ${ }^{141}$, M. Trzebinski ${ }^{38}$, A. Trzupek ${ }^{38}$, C. Tsarouchas ${ }^{29}$, J.C-L. Tseng ${ }^{117}$, M. Tsiakiris ${ }^{104}$, P.V. Tsiareshka ${ }^{89}$, D. Tsionou ${ }^{4}$,ai, G. Tsipolitis ${ }^{9}$, S. Tsiskaridze ${ }^{11}$, V. Tsiskaridze ${ }^{47}$, E.G. Tskhadadze ${ }^{50 a}$, I.I. Tsukerman ${ }^{94}$, V. Tsulaia ${ }^{14}$, J.-W. Tsung ${ }^{20}$, S. Tsuno ${ }^{64}$, D. Tsybychev ${ }^{147}$, A. Tua ${ }^{138}$, A. Tudorache ${ }^{25 a}$, V. Tudorache ${ }^{25 a}$, J.M. Tuggle ${ }^{30}$, M. Turala ${ }^{38}$, D. Turecek ${ }^{126}$, I. Turk Cakir ${ }^{3 e}$, E. Turlay ${ }^{104}$, R. Turra ${ }^{88 a, 88 b}$, P.M. Tuts ${ }^{34}$, A. Tykhonov ${ }^{73}$, M. Tylmad $^{145 a, 145 b}$, M. Tyndel ${ }^{128}$, G. Tzanakos ${ }^{8}$, K. Uchida ${ }^{20}$, I. Ueda ${ }^{154}$, R. Ueno ${ }^{28}$, M. Ugland ${ }^{13}$, M. Uhlenbrock ${ }^{20}$, M. Uhrmacher ${ }^{53}$, F. Ukegawa ${ }^{159}$, G. Unal ${ }^{29}$, A. Undrus $^{24}$, G. Unel ${ }^{162}$, Y. Unno ${ }^{64}$, D. Urbaniec ${ }^{34}$, G. Usai ${ }^{7}$, M. Uslenghi ${ }^{118 a, 118 b}$, L. Vacavant ${ }^{82}$, V. Vacek ${ }^{126}$, B. Vachon ${ }^{84}$, S. Vahsen ${ }^{14}$, J. Valenta ${ }^{124}$, S. Valentinetti ${ }^{19 a, 19 b}$, A. Valero ${ }^{166}$, S. Valkar ${ }^{125}$, E. Valladolid Gallego ${ }^{166}$, S. Vallecorsa ${ }^{151}$, J.A. Valls Ferrer ${ }^{166}$, P.C. Van Der Deij ${ }^{104}$, R. van der Geer ${ }^{104}$, H. van der Graaf ${ }^{104}$, R. Van Der Leeuw ${ }^{104}$, E. van der Poel ${ }^{104}$, D. van der $\operatorname{Ster}^{29}$, N. van Eldik $^{29}$, P. van Gemmeren ${ }^{5}$, I. van Vulpen ${ }^{104}$, M. Vanadia ${ }^{98}$, W. Vandelli ${ }^{29}$, A. Vaniachine ${ }^{5}$, P. Vankov ${ }^{41}$, F. Vannucci ${ }^{77}$, R. Vari ${ }^{131 a}$, T. Varol ${ }^{83}$, D. Varouchas ${ }^{14}$, A. Vartapetian ${ }^{7}$, K.E. Varvell ${ }^{149}$, V.I. Vassilakopoulos ${ }^{55}$, F. Vazeille ${ }^{33}$, T. Vazquez Schroeder ${ }^{53}$, G. Vegni ${ }^{88 a}, 88 b$, J.J. Veillet ${ }^{114}$, F. Veloso ${ }^{123 a}$, R. Veness $^{29}$, S. Veneziano ${ }^{131 a}$, A. Ventura $^{71 a, 71 b}$, D. Ventura ${ }^{83}$, M. Venturi ${ }^{47}$, N. Venturi ${ }^{157}$, V. Vercesi ${ }^{118 a}$, M. Verducci ${ }^{137}$, W. Verkerke ${ }^{104}$, J.C. Vermeulen ${ }^{104}$, A. Vest ${ }^{43}$, M.C. Vetterli ${ }^{141, d}$, I. Vichou ${ }^{164}$, T. Vickey ${ }^{144 b, a j}$, O.E. Vickey Boeriu ${ }^{144 b}$, G.H.A. Viehhauser ${ }^{117}$, S. Viel ${ }^{167}$, M. Villa ${ }^{19 a, 19 b}$, M. Villaplana Perez ${ }^{166}$, E. Vilucchi ${ }^{46}$, M.G. Vincter ${ }^{28}$, E. Vinek ${ }^{29}$, V.B. Vinogradov ${ }^{63}$, M. Virchaux ${ }^{135, *}$, J. Virzi ${ }^{14}$, O. Vitells ${ }^{171}$, M. Viti ${ }^{41}$, I. Vivarelli ${ }^{47}$, F. Vives Vaque ${ }^{2}$, S. Vlachos ${ }^{9}$, D. Vladoiu ${ }^{97}$, M. Vlasak ${ }^{126}$, A. Vogel ${ }^{20}$, P. Vokac ${ }^{126}$, G. Volpi ${ }^{46}$, M. Volpi ${ }^{85}$, G. Volpini ${ }^{88 a}$, H. von der Schmitt $^{98}$, H. von Radziewski ${ }^{47}$, E. von Toerne ${ }^{20}$, V. Vorobel $^{125}$, V. Vorwerk ${ }^{11}$, M. Vos ${ }^{166}$, R. Voss ${ }^{29}$, T.T. Voss ${ }^{174}$, J.H. Vossebeld ${ }^{72}$, N. Vranjes ${ }^{135}$, M. Vranjes Milosavljevic ${ }^{104}$, V. Vrba ${ }^{124}$, M. Vreeswijk ${ }^{104}$, T. Vu Anh ${ }^{47}$, R. Vuillermet ${ }^{29}$, I. Vukotic ${ }^{30}$, W. Wagner ${ }^{174}$, P. Wagner ${ }^{119}$, H. Wahlen ${ }^{174}$, S. Wahrmund ${ }^{43}$, J. Wakabayashi ${ }^{100}$, S. Walch ${ }^{86}$, J. Walder ${ }^{70}$, R. Walker ${ }^{97}$, W. Walkowiak ${ }^{140}$, R. Wall ${ }^{175}$, P. Waller ${ }^{72}$, B. Walsh ${ }^{175}$, C. Wang ${ }^{44}$, H. Wang ${ }^{172}$, H. Wang ${ }^{32 b, a k}$, J. Wang ${ }^{150}$, J. Wang ${ }^{54}$, R. Wang ${ }^{102}$, S.M. Wang ${ }^{150}$, T. Wang ${ }^{20}$, A. Warburton ${ }^{84}$, C.P. Ward ${ }^{27}$, M. Warsinsky ${ }^{47}$, A. Washbrook ${ }^{45}$, C. Wasicki ${ }^{41}$, I. Watanabe ${ }^{65}$, P.M. Watkins ${ }^{17}$, A.T. Watson $^{17}$, I.J. Watson ${ }^{149}$, M.F. Watson ${ }^{17}$, G. Watts ${ }^{137}$, S. Watts ${ }^{81}$, A.T. Waugh ${ }^{149}$, B.M. Waugh ${ }^{76}$, M.S. Weber ${ }^{16}$, P. Weber ${ }^{53}$, A.R. Weidberg ${ }^{117}$, P. Weigell ${ }^{98}$, J. Weingarten ${ }^{53}$, C. Weiser ${ }^{47}$, H. Wellenstein ${ }^{22}$, P.S. Wells ${ }^{29}$, T. Wenaus ${ }^{24}$, D. Wendland ${ }^{15}$, Z. Weng ${ }^{150, w}$, T. Wengler ${ }^{29}$, S. Wenig ${ }^{29}$, N. Wermes ${ }^{20}$, M. Werner ${ }^{47}$, P. Werner ${ }^{29}$, M. Werth ${ }^{162}$, M. Wessels ${ }^{57 a}$, J. Wetter ${ }^{160}$, C. Weydert ${ }^{54}$, K. Whalen ${ }^{28}$, S.J. Wheeler-Ellis ${ }^{162}$, A. White ${ }^{7}$, M.J. White ${ }^{85}$, S. White ${ }^{121 a, 121 b}$, S.R. Whitehead ${ }^{117}$, D. Whiteson $^{162}$, D. Whittington ${ }^{59}$, F. Wicek ${ }^{114}$, D. Wicke ${ }^{174}$, F.J. Wickens ${ }^{128}$, W. Wiedenmann ${ }^{172}$, M. Wielers ${ }^{128}$, P. Wienemann ${ }^{20}$, C. Wiglesworth ${ }^{74}$, L.A.M. Wiik-Fuchs ${ }^{47}$, P.A. Wijeratne ${ }^{76}$, A. Wildauer ${ }^{98}$, M.A. Wildt ${ }^{41, \mathrm{~s}}$, I. Wilhelm ${ }^{125}$, H.G. Wilkens ${ }^{29}$, J.Z. Will ${ }^{97}$, E. Williams ${ }^{34}$, H.H. Williams ${ }^{119}$, W. Willis ${ }^{34}$, S. Willocq ${ }^{83}$, J.A. Wilson ${ }^{17}$, M.G. Wilson ${ }^{142}$, A. Wilson ${ }^{86}$, I. Wingerter-Seez ${ }^{4}$, S. Winkelmann ${ }^{47}$, F. Winklmeier ${ }^{29}$, M. Wittgen ${ }^{142}$, S.J. Wollstadt ${ }^{80}$, M.W. Wolter ${ }^{38}$, H. Wolters ${ }^{123 a, h}$, W.C. Wong ${ }^{40}$, G. Wooden ${ }^{86}$, B.K. Wosiek ${ }^{38}$, J. Wotschack ${ }^{29}$, M.J. Woudstra ${ }^{81}$, K.W. Wozniak ${ }^{38}$, K. Wraight ${ }^{52}$, C. Wright ${ }^{52}$, M. Wright ${ }^{52}$, B. Wrona ${ }^{72}$, S.L. Wu ${ }^{172}$, X. Wu ${ }^{48}$, Y. Wu ${ }^{32 b, a l}$, E. Wulf ${ }^{34}$, B.M. Wynne ${ }^{45}$, S. Xella ${ }^{35}$, M. Xiao ${ }^{135}$, S. Xie ${ }^{47}$, C. $\mathrm{Xu}^{32 \mathrm{~b}, \mathrm{z}}$, D. Xu ${ }^{138}$, B. Yabsley ${ }^{149}$, S. Yacoob ${ }^{144 \mathrm{~b}}$, M. Yamada ${ }^{64}$, H. Yamaguchi ${ }^{154}$, A. Yamamoto ${ }^{64}$, K. Yamamoto ${ }^{62}$, S. Yamamoto ${ }^{154}$, T. Yamamura ${ }^{154}$, T. Yamanaka ${ }^{154}$, J. Yamaoka ${ }^{44}$, T. Yamazaki ${ }^{154}$, Y. Yamazaki ${ }^{65}$, Z. Yan $^{21}$, H. Yang $^{86}$, U.K. Yang ${ }^{81}$, Y. Yang ${ }^{59}$, Z. Yang ${ }^{145 a, 145 b}$, S. Yanush ${ }^{90}$, L. Yao ${ }^{32 a}$, Y. Yao ${ }^{14}$, Y. Yasu ${ }^{64}$, G.V. Ybeles Smit ${ }^{129}$, J. Ye ${ }^{39}$, S. Ye ${ }^{24}$, M. Yilmaz ${ }^{3 c}$, R. Yoosoofmiya ${ }^{122}$, K. Yorita ${ }^{170}$, R. Yoshida ${ }^{5}$, C. Young ${ }^{142}$, C.J. Young ${ }^{117}$, S. Youssef ${ }^{21}$, D. Yu $^{24}$, J. Yu ${ }^{7}$, J. Yu ${ }^{111}$, L. Yuan ${ }^{65}$, A. Yurkewicz ${ }^{105}$, M. Byszewski ${ }^{29}$, B. Zabinski ${ }^{38}$, R. Zaidan ${ }^{61}$, A.M. Zaitsev ${ }^{127}$, Z. Zajacova $^{29}$, L. Zanello ${ }^{131 \mathrm{a}, 131 \mathrm{~b}}$, A. Zaytsev ${ }^{106}$, C. Zeitnitz ${ }^{174}$, M. Zeman ${ }^{124}$, A. Zemla ${ }^{38}$, C. Zendler ${ }^{20}$, O. Zenin ${ }^{127}$, T. Ženiš ${ }^{143 a}$, 
Z. Zinonos ${ }^{121 a, 121 b}$, S. Zenz ${ }^{14}$, D. Zerwas ${ }^{114}$, G. Zevi della Porta ${ }^{56}$, Z. Zhan ${ }^{32 \mathrm{~d}}$, D. Zhang ${ }^{32 b, a k}$, H. Zhang ${ }^{87}$, J. Zhang ${ }^{5}$, X. Zhang ${ }^{32 d}$, Z. Zhang ${ }^{114}$, L. Zhao ${ }^{107}$, T. Zhao ${ }^{137}$, Z. Zhao ${ }^{32 b}$, A. Zhemchugov ${ }^{63}$, J. Zhong ${ }^{117}$, B. Zhou ${ }^{86}$, N. Zhou ${ }^{162}$, Y. Zhou ${ }^{150}$, C.G. Zhu ${ }^{32 d}$, H. Zhu ${ }^{41}$, J. Zhu ${ }^{86}$, Y. Zhu ${ }^{32 b}$, X. Zhuang ${ }^{97}$, V. Zhuravlov ${ }^{98}$, D. Zieminska ${ }^{59}$, N.I. Zimin ${ }^{63}$, R. Zimmermann ${ }^{20}$, S. Zimmermann ${ }^{20}$, S. Zimmermann ${ }^{47}$, M. Ziolkowski ${ }^{140}$, R. Zitoun ${ }^{4}$, L. Živković ${ }^{34}$, V.V. Zmouchko ${ }^{127, *}$, G. Zobernig ${ }^{172}$, A. Zoccoli ${ }^{19 a, 19 b}$, M. zur Nedden ${ }^{15}$, V. Zutshi ${ }^{105}$, L. Zwalinski ${ }^{29}$

${ }^{1}$ Physics Department, SUNY Albany, Albany NY, United States of America

${ }^{2}$ Department of Physics, University of Alberta, Edmonton AB, Canada

${ }^{3(\mathrm{a})}$ Department of Physics, Ankara University, Ankara; ${ }^{(\mathrm{b})}$ Department of Physics, Dumlupinar University, Kutahya;

${ }^{(c)}$ Department of Physics, Gazi University, Ankara; ${ }^{(d)}$ Division of Physics, TOBB University of Economics and

Technology, Ankara; ${ }^{(\mathrm{e})}$ Turkish Atomic Energy Authority, Ankara, Turkey

${ }^{4}$ LAPP, CNRS/IN2P3 and Université de Savoie, Annecy-le-Vieux, France

${ }^{5}$ High Energy Physics Division, Argonne National Laboratory, Argonne IL, United States of America

${ }^{6}$ Department of Physics, University of Arizona, Tucson AZ, United States of America

${ }^{7}$ Department of Physics, The University of Texas at Arlington, Arlington TX, United States of America

${ }^{8}$ Physics Department, University of Athens, Athens, Greece

${ }^{9}$ Physics Department, National Technical University of Athens, Zografou, Greece

${ }^{10}$ Institute of Physics, Azerbaijan Academy of Sciences, Baku, Azerbaijan

${ }^{11}$ Institut de Física d'Altes Energies and Departament de Física de la Universitat Autònoma de Barcelona and ICREA, Barcelona, Spain

12(a) Institute of Physics, University of Belgrade, Belgrade; (b) Vinca Institute of Nuclear Sciences, University of Belgrade,

Belgrade, Serbia

${ }^{13}$ Department for Physics and Technology, University of Bergen, Bergen, Norway

${ }^{14}$ Physics Division, Lawrence Berkeley National Laboratory and University of California, Berkeley CA, United States of America

${ }^{15}$ Department of Physics, Humboldt University, Berlin, Germany

${ }^{16}$ Albert Einstein Center for Fundamental Physics and Laboratory for High Energy Physics, University of Bern, Bern, Switzerland

${ }^{17}$ School of Physics and Astronomy, University of Birmingham, Birmingham, United Kingdom

18(a) Department of Physics, Bogazici University, Istanbul; ${ }^{(b)}$ Division of Physics, Dogus University, Istanbul;

${ }^{(c)}$ Department of Physics Engineering, Gaziantep University, Gaziantep; ${ }^{(d)}$ Department of Physics, Istanbul Technical University, Istanbul, Turkey

19(a) INFN Sezione di Bologna; ${ }^{(b)}$ Dipartimento di Fisica, Università di Bologna, Bologna, Italy

${ }^{20}$ Physikalisches Institut, University of Bonn, Bonn, Germany

${ }^{21}$ Department of Physics, Boston University, Boston MA, United States of America

${ }^{22}$ Department of Physics, Brandeis University, Waltham MA, United States of America

23(a) Universidade Federal do Rio De Janeiro COPPE/EE/IF, Rio de Janeiro; ${ }^{(b)}$ Federal University of Juiz de Fora (UFJF), Juiz de Fora; ${ }^{\left({ }^{c}\right)}$ Federal University of Sao Joao del Rei (UFSJ), Sao Joao del Rei; ${ }^{(d)}$ Instituto de Fisica, Universidade de Sao Paulo, Sao Paulo, Brazil

${ }^{24}$ Physics Department, Brookhaven National Laboratory, Upton NY, United States of America

25(a) National Institute of Physics and Nuclear Engineering, Bucharest; ${ }^{(b)}$ University Politehnica Bucharest, Bucharest;

${ }^{(c)}$ West University in Timisoara, Timisoara, Romania

${ }^{26}$ Departamento de Física, Universidad de Buenos Aires, Buenos Aires, Argentina

${ }^{27}$ Cavendish Laboratory, University of Cambridge, Cambridge, United Kingdom

${ }^{28}$ Department of Physics, Carleton University, Ottawa ON, Canada

${ }^{29}$ CERN, Geneva, Switzerland

${ }^{30}$ Enrico Fermi Institute, University of Chicago, Chicago IL, United States of America

31(a) Departamento de Física, Pontificia Universidad Católica de Chile, Santiago; ${ }^{(b)}$ Departamento de Física, Universidad Técnica Federico Santa María, Valparaíso, Chile

32(a) Institute of High Energy Physics, Chinese Academy of Sciences, Beijing; ${ }^{(b)}$ Department of Modern Physics, University of Science and Technology of China, Anhui; ${ }^{(c)}$ Department of Physics, Nanjing University, Jiangsu; ${ }^{(d)}$ School of Physics, Shandong University, Shandong, China

${ }^{33}$ Laboratoire de Physique Corpusculaire, Clermont Université and Université Blaise Pascal and CNRS/IN2P3, Aubiere Cedex, France 
${ }^{34}$ Nevis Laboratory, Columbia University, Irvington NY, United States of America

${ }^{35}$ Niels Bohr Institute, University of Copenhagen, Kobenhavn, Denmark

36(a) INFN Gruppo Collegato di Cosenza; ${ }^{(b)}$ Dipartimento di Fisica, Università della Calabria, Arcavata di Rende, Italy

${ }^{37}$ AGH University of Science and Technology, Faculty of Physics and Applied Computer Science, Krakow, Poland

${ }^{38}$ The Henryk Niewodniczanski Institute of Nuclear Physics, Polish Academy of Sciences, Krakow, Poland

${ }^{39}$ Physics Department, Southern Methodist University, Dallas TX, United States of America

${ }^{40}$ Physics Department, University of Texas at Dallas, Richardson TX, United States of America

${ }^{41}$ DESY, Hamburg and Zeuthen, Germany

${ }^{42}$ Institut für Experimentelle Physik IV, Technische Universität Dortmund, Dortmund, Germany

${ }^{43}$ Institut für Kern-und Teilchenphysik, Technical University Dresden, Dresden, Germany

${ }^{44}$ Department of Physics, Duke University, Durham NC, United States of America

${ }^{45}$ SUPA - School of Physics and Astronomy, University of Edinburgh, Edinburgh, United Kingdom

${ }^{46}$ INFN Laboratori Nazionali di Frascati, Frascati, Italy

${ }^{47}$ Fakultät für Mathematik und Physik, Albert-Ludwigs-Universität, Freiburg, Germany

${ }^{48}$ Section de Physique, Université de Genève, Geneva, Switzerland

49(a) INFN Sezione di Genova; ${ }^{(b)}$ Dipartimento di Fisica, Università di Genova, Genova, Italy

${ }^{50(a)}$ E. Andronikashvili Institute of Physics, Tbilisi State University, Tbilisi; ${ }^{(b)}$ High Energy Physics Institute, Tbilisi State University, Tbilisi, Georgia

${ }^{51}$ II Physikalisches Institut, Justus-Liebig-Universität Giessen, Giessen, Germany

${ }^{52}$ SUPA - School of Physics and Astronomy, University of Glasgow, Glasgow, United Kingdom

${ }^{53}$ II Physikalisches Institut, Georg-August-Universität, Göttingen, Germany

${ }^{54}$ Laboratoire de Physique Subatomique et de Cosmologie, Université Joseph Fourier and CNRS/IN2P3 and Institut National Polytechnique de Grenoble, Grenoble, France

${ }^{55}$ Department of Physics, Hampton University, Hampton VA, United States of America

${ }^{56}$ Laboratory for Particle Physics and Cosmology, Harvard University, Cambridge MA, United States of America

${ }^{57(a)}$ Kirchhoff-Institut für Physik, Ruprecht-Karls-Universität Heidelberg, Heidelberg; ${ }^{(b)}$ Physikalisches Institut,

Ruprecht-Karls-Universität Heidelberg, Heidelberg; ${ }^{(c)}$ ZITI Institut für technische Informatik,

Ruprecht-Karls-Universität Heidelberg, Mannheim, Germany

${ }^{58}$ Faculty of Applied Information Science, Hiroshima Institute of Technology, Hiroshima, Japan

${ }^{59}$ Department of Physics, Indiana University, Bloomington IN, United States of America

${ }^{60}$ Institut für Astro-und Teilchenphysik, Leopold-Franzens-Universität, Innsbruck, Austria

${ }^{61}$ University of Iowa, Iowa City IA, United States of America

${ }^{62}$ Department of Physics and Astronomy, Iowa State University, Ames IA, United States of America

${ }^{63}$ Joint Institute for Nuclear Research, JINR Dubna, Dubna, Russia

${ }^{64}$ KEK, High Energy Accelerator Research Organization, Tsukuba, Japan

${ }^{65}$ Graduate School of Science, Kobe University, Kobe, Japan

${ }^{66}$ Faculty of Science, Kyoto University, Kyoto, Japan

${ }^{67}$ Kyoto University of Education, Kyoto, Japan

${ }^{68}$ Department of Physics, Kyushu University, Fukuoka, Japan

${ }^{69}$ Instituto de Física La Plata, Universidad Nacional de La Plata and CONICET, La Plata, Argentina

${ }^{70}$ Physics Department, Lancaster University, Lancaster, United Kingdom

71(a) INFN Sezione di Lecce; ${ }^{(b)}$ Dipartimento di Matematica e Fisica, Università del Salento, Lecce, Italy

${ }^{72}$ Oliver Lodge Laboratory, University of Liverpool, Liverpool, United Kingdom

${ }^{73}$ Department of Physics, Jožef Stefan Institute and University of Ljubljana, Ljubljana, Slovenia

${ }^{74}$ School of Physics and Astronomy, Queen Mary University of London, London, United Kingdom

${ }^{75}$ Department of Physics, Royal Holloway University of London, Surrey, United Kingdom

${ }^{76}$ Department of Physics and Astronomy, University College London, London, United Kingdom

${ }^{77}$ Laboratoire de Physique Nucléaire et de Hautes Energies, UPMC and Université Paris-Diderot and CNRS/IN2P3, Paris, France

${ }^{78}$ Fysiska institutionen, Lunds universitet, Lund, Sweden

${ }^{79}$ Departamento de Fisica Teorica C-15, Universidad Autonoma de Madrid, Madrid, Spain

${ }^{80}$ Institut für Physik, Universität Mainz, Mainz, Germany

${ }^{81}$ School of Physics and Astronomy, University of Manchester, Manchester, United Kingdom 
${ }^{82} \mathrm{CPPM}$, Aix-Marseille Université and CNRS/IN2P3, Marseille, France

${ }^{83}$ Department of Physics, University of Massachusetts, Amherst MA, United States of America

${ }^{84}$ Department of Physics, McGill University, Montreal QC, Canada

${ }^{85}$ School of Physics, University of Melbourne, Victoria, Australia

${ }^{86}$ Department of Physics, The University of Michigan, Ann Arbor MI, United States of America

${ }^{87}$ Department of Physics and Astronomy, Michigan State University, East Lansing MI, United States of America

${ }^{88(a)}$ INFN Sezione di Milano; ${ }^{(b)}$ Dipartimento di Fisica, Università di Milano, Milano, Italy

${ }^{89}$ B.I. Stepanov Institute of Physics, National Academy of Sciences of Belarus, Minsk, Republic of Belarus

${ }^{90}$ National Scientific and Educational Centre for Particle and High Energy Physics, Minsk, Republic of Belarus

${ }^{91}$ Department of Physics, Massachusetts Institute of Technology, Cambridge MA, United States of America

${ }^{92}$ Group of Particle Physics, University of Montreal, Montreal QC, Canada

${ }^{93}$ P.N. Lebedev Institute of Physics, Academy of Sciences, Moscow, Russia

${ }^{94}$ Institute for Theoretical and Experimental Physics (ITEP), Moscow, Russia

${ }^{95}$ Moscow Engineering and Physics Institute (MEPhI), Moscow, Russia

${ }^{96}$ Skobeltsyn Institute of Nuclear Physics, Lomonosov Moscow State University, Moscow, Russia

${ }^{97}$ Fakultät für Physik, Ludwig-Maximilians-Universität München, München, Germany

${ }^{98}$ Max-Planck-Institut für Physik (Werner-Heisenberg-Institut), München, Germany

${ }^{99}$ Nagasaki Institute of Applied Science, Nagasaki, Japan

${ }^{100}$ Graduate School of Science and Kobayashi-Maskawa Institute, Nagoya University, Nagoya, Japan

101 (a) INFN Sezione di Napoli; ${ }^{(b)}$ Dipartimento di Scienze Fisiche, Università di Napoli, Napoli, Italy

${ }^{102}$ Department of Physics and Astronomy, University of New Mexico, Albuquerque NM, United States of America

${ }^{103}$ Institute for Mathematics, Astrophysics and Particle Physics, Radboud University Nijmegen/Nikhef, Nijmegen, Netherlands

${ }^{104}$ Nikhef National Institute for Subatomic Physics and University of Amsterdam, Amsterdam, Netherlands

${ }^{105}$ Department of Physics, Northern Illinois University, DeKalb IL, United States of America

${ }^{106}$ Budker Institute of Nuclear Physics, SB RAS, Novosibirsk, Russia

${ }^{107}$ Department of Physics, New York University, New York NY, United States of America

${ }^{108}$ Ohio State University, Columbus OH, United States of America

${ }^{109}$ Faculty of Science, Okayama University, Okayama, Japan

${ }^{110}$ Homer L. Dodge Department of Physics and Astronomy, University of Oklahoma, Norman OK, United States of America

${ }^{111}$ Department of Physics, Oklahoma State University, Stillwater OK, United States of America

${ }^{112}$ Palacký University, RCPTM, Olomouc, Czech Republic

${ }^{113}$ Center for High Energy Physics, University of Oregon, Eugene OR, United States of America

${ }^{114}$ LAL, Université Paris-Sud and CNRS/IN2P3, Orsay, France

${ }^{115}$ Graduate School of Science, Osaka University, Osaka, Japan

${ }^{116}$ Department of Physics, University of Oslo, Oslo, Norway

${ }^{117}$ Department of Physics, Oxford University, Oxford, United Kingdom

${ }^{118(a)}$ INFN Sezione di Pavia; ${ }^{(b)}$ Dipartimento di Fisica, Università di Pavia, Pavia, Italy

${ }^{119}$ Department of Physics, University of Pennsylvania, Philadelphia PA, United States of America

${ }^{120}$ Petersburg Nuclear Physics Institute, Gatchina, Russia

121 (a) INFN Sezione di Pisa; ${ }^{(b)}$ Dipartimento di Fisica E. Fermi, Università di Pisa, Pisa, Italy

${ }^{122}$ Department of Physics and Astronomy, University of Pittsburgh, Pittsburgh PA, United States of America

${ }^{123(a)}$ Laboratorio de Instrumentacao e Fisica Experimental de Particulas - LIP, Lisboa, Portugal; ${ }^{(b)}$ Departamento de Fisica

Teorica y del Cosmos and CAFPE, Universidad de Granada, Granada, Spain

${ }^{124}$ Institute of Physics, Academy of Sciences of the Czech Republic, Praha, Czech Republic

${ }^{125}$ Faculty of Mathematics and Physics, Charles University in Prague, Praha, Czech Republic

${ }^{126}$ Czech Technical University in Prague, Praha, Czech Republic

${ }^{127}$ State Research Center Institute for High Energy Physics, Protvino, Russia

${ }^{128}$ Particle Physics Department, Rutherford Appleton Laboratory, Didcot, United Kingdom

${ }^{129}$ Physics Department, University of Regina, Regina SK, Canada

${ }^{130}$ Ritsumeikan University, Kusatsu, Shiga, Japan

131(a) INFN Sezione di Roma I; ${ }^{\left({ }^{b}\right)}$ Dipartimento di Fisica, Università La Sapienza, Roma, Italy 
132(a) INFN Sezione di Roma Tor Vergata; ${ }^{(b)}$ Dipartimento di Fisica, Università di Roma Tor Vergata, Roma, Italy 133(a) INFN Sezione di Roma Tre; ${ }^{\left({ }^{b}\right)}$ Dipartimento di Fisica, Università Roma Tre, Roma, Italy

${ }^{134(a)}$ Faculté des Sciences Ain Chock, Réseau Universitaire de Physique des Hautes Energies - Université Hassan II, Casablanca; ${ }^{(b)}$ Centre National de l'Energie des Sciences Techniques Nucleaires, Rabat; ${ }^{(c)}$ Faculté des Sciences

Semlalia, Université Cadi Ayyad, LPHEA-Marrakech; ${ }^{(d)}$ Faculté des Sciences, Université Mohamed Premier and

LPTPM, Oujda; ${ }^{(e)}$ Faculté des sciences, Université Mohammed V-Agdal, Rabat, Morocco

${ }^{135}$ DSM/IRFU (Institut de Recherches sur les Lois Fondamentales de l'Univers), CEA Saclay (Commissariat a l'Energie

Atomique), Gif-sur-Yvette, France

${ }^{136}$ Santa Cruz Institute for Particle Physics, University of California Santa Cruz, Santa Cruz CA, United States of America

${ }^{137}$ Department of Physics, University of Washington, Seattle WA, United States of America

${ }^{138}$ Department of Physics and Astronomy, University of Sheffield, Sheffield, United Kingdom

${ }^{139}$ Department of Physics, Shinshu University, Nagano, Japan

${ }^{140}$ Fachbereich Physik, Universität Siegen, Siegen, Germany

${ }^{141}$ Department of Physics, Simon Fraser University, Burnaby BC, Canada

${ }^{142}$ SLAC National Accelerator Laboratory, Stanford CA, United States of America

${ }^{143(a)}$ Faculty of Mathematics, Physics \& Informatics, Comenius University, Bratislava; ${ }^{(b)}$ Department of Subnuclear

Physics, Institute of Experimental Physics of the Slovak Academy of Sciences, Kosice, Slovak Republic

${ }^{144(a)}$ Department of Physics, University of Johannesburg, Johannesburg; ${ }^{\left({ }^{b}\right)}$ School of Physics, University of the

Witwatersrand, Johannesburg, South Africa

145(a) Department of Physics, Stockholm University; ${ }^{(b)}$ The Oskar Klein Centre, Stockholm, Sweden

${ }^{146}$ Physics Department, Royal Institute of Technology, Stockholm, Sweden

${ }^{147}$ Departments of Physics \& Astronomy and Chemistry, Stony Brook University, Stony Brook NY, United States of

America

${ }^{148}$ Department of Physics and Astronomy, University of Sussex, Brighton, United Kingdom

${ }^{149}$ School of Physics, University of Sydney, Sydney, Australia

${ }^{150}$ Institute of Physics, Academia Sinica, Taipei, Taiwan

${ }^{151}$ Department of Physics, Technion: Israel Institute of Technology, Haifa, Israel

${ }^{152}$ Raymond and Beverly Sackler School of Physics and Astronomy, Tel Aviv University, Tel Aviv, Israel

${ }^{153}$ Department of Physics, Aristotle University of Thessaloniki, Thessaloniki, Greece

${ }^{154}$ International Center for Elementary Particle Physics and Department of Physics, The University of Tokyo, Tokyo, Japan

${ }^{155}$ Graduate School of Science and Technology, Tokyo Metropolitan University, Tokyo, Japan

${ }^{156}$ Department of Physics, Tokyo Institute of Technology, Tokyo, Japan

${ }^{157}$ Department of Physics, University of Toronto, Toronto ON, Canada

158(a) TRIUMF, Vancouver BC; ${ }^{(\mathrm{b})}$ Department of Physics and Astronomy, York University, Toronto ON, Canada

${ }^{159}$ Institute of Pure and Applied Sciences, University of Tsukuba, 1-1-1 Tennodai, Tsukuba, Ibaraki 305-8571, Japan

${ }^{160}$ Science and Technology Center, Tufts University, Medford MA, United States of America

${ }^{161}$ Centro de Investigaciones, Universidad Antonio Narino, Bogota, Colombia

${ }^{162}$ Department of Physics and Astronomy, University of California Irvine, Irvine CA, United States of America

${ }^{163(a)}$ INFN Gruppo Collegato di Udine, Udine; ${ }^{(b)}$ ICTP, Trieste; ${ }^{(c)}$ Dipartimento di Chimica, Fisica e Ambiente, Università

di Udine, Udine, Italy

${ }^{164}$ Department of Physics, University of Illinois, Urbana IL, United States of America

${ }^{165}$ Department of Physics and Astronomy, University of Uppsala, Uppsala, Sweden

${ }^{166}$ Instituto de Física Corpuscular (IFIC) and Departamento de Física Atómica, Molecular y Nuclear and Departamento de Ingeniería Electrónica and Instituto de Microelectrónica de Barcelona (IMB-CNM), University of Valencia and CSIC, Valencia, Spain

${ }^{167}$ Department of Physics, University of British Columbia, Vancouver BC, Canada

${ }^{168}$ Department of Physics and Astronomy, University of Victoria, Victoria BC, Canada

${ }^{169}$ Department of Physics, University of Warwick, Coventry, United Kingdom

${ }^{170}$ Waseda University, Tokyo, Japan

${ }^{171}$ Department of Particle Physics, The Weizmann Institute of Science, Rehovot, Israel

${ }^{172}$ Department of Physics, University of Wisconsin, Madison WI, United States of America

${ }^{173}$ Fakultät für Physik und Astronomie, Julius-Maximilians-Universität, Würzburg, Germany

${ }^{174}$ Fachbereich C Physik, Bergische Universität Wuppertal, Wuppertal, Germany 
${ }^{175}$ Department of Physics, Yale University, New Haven CT, United States of America

${ }^{176}$ Yerevan Physics Institute, Yerevan, Armenia

${ }^{177}$ Domaine scientifique de la Doua, Centre de Calcul CNRS/IN2P3, Villeurbanne Cedex, France

${ }^{a}$ Also at Laboratorio de Instrumentacao e Fisica Experimental de Particulas - LIP, Lisboa, Portugal

${ }^{\mathrm{b}}$ Also at Faculdade de Ciencias and CFNUL, Universidade de Lisboa, Lisboa, Portugal

${ }^{\mathrm{c}}$ Also at Particle Physics Department, Rutherford Appleton Laboratory, Didcot, United Kingdom

${ }^{\mathrm{d}}$ Also at TRIUMF, Vancouver BC, Canada

${ }^{\mathrm{e}}$ Also at Department of Physics, California State University, Fresno CA, United States of America

${ }^{\mathrm{f}}$ Also at Novosibirsk State University, Novosibirsk, Russia

${ }^{\mathrm{g}}$ Also at Fermilab, Batavia IL, United States of America

h Also at Department of Physics, University of Coimbra, Coimbra, Portugal

${ }^{\mathrm{i}}$ Also at Department of Physics, UASLP, San Luis Potosi, Mexico

${ }^{\mathrm{j}}$ Also at Università di Napoli Parthenope, Napoli, Italy

${ }^{\mathrm{k}}$ Also at Institute of Particle Physics (IPP), Canada

${ }^{1}$ Also at Department of Physics, Middle East Technical University, Ankara, Turkey

${ }^{m}$ Also at Louisiana Tech University, Ruston LA, United States of America

${ }^{\mathrm{n}}$ Also at Dep Fisica and CEFITEC of Faculdade de Ciencias e Tecnologia, Universidade Nova de Lisboa, Caparica, Portugal

${ }^{\circ}$ Also at Department of Physics and Astronomy, University College London, London, United Kingdom

PAlso at Group of Particle Physics, University of Montreal, Montreal QC, Canada

${ }^{\mathrm{q}}$ Also at Department of Physics, University of Cape Town, Cape Town, South Africa

${ }^{\mathrm{r}}$ Also at Institute of Physics, Azerbaijan Academy of Sciences, Baku, Azerbaijan

${ }^{\mathrm{s}}$ Also at Institut für Experimentalphysik, Universität Hamburg, Hamburg, Germany

${ }^{t}$ Also at Manhattan College, New York NY, United States of America

${ }^{u}$ Also at School of Physics, Shandong University, Shandong, China

${ }^{\mathrm{v}}$ Also at CPPM, Aix-Marseille Université and CNRS/IN2P3, Marseille, France

${ }^{w}$ Also at School of Physics and Engineering, Sun Yat-sen University, Guanzhou, China

${ }^{x}$ Also at Academia Sinica Grid Computing, Institute of Physics, Academia Sinica, Taipei, Taiwan

y Also at Dipartimento di Fisica, Università La Sapienza, Roma, Italy

${ }^{\mathrm{z}}$ Also at DSM/IRFU (Institut de Recherches sur les Lois Fondamentales de l'Univers), CEA Saclay (Commissariat a l'Energie Atomique), Gif-sur-Yvette, France

aa Also at Section de Physique, Université de Genève, Geneva, Switzerland

ab Also at Departamento de Fisica, Universidade de Minho, Braga, Portugal

${ }^{a c}$ Also at Department of Physics and Astronomy, University of South Carolina, Columbia SC, United States of America

${ }^{a d}$ Also at Institute for Particle and Nuclear Physics, Wigner Research Centre for Physics, Budapest, Hungary

${ }^{a e}$ Also at California Institute of Technology, Pasadena CA, United States of America

${ }^{a f}$ Also at Institute of Physics, Jagiellonian University, Krakow, Poland

${ }^{\text {ag }}$ Also at LAL, Université Paris-Sud and CNRS/IN2P3, Orsay, France

${ }^{\text {ah }}$ Also at Nevis Laboratory, Columbia University, Irvington NY, United States of America

ai Also at Department of Physics and Astronomy, University of Sheffield, Sheffield, United Kingdom

aj Also at Department of Physics, Oxford University, Oxford, United Kingdom

${ }^{\mathrm{ak}}$ Also at Institute of Physics, Academia Sinica, Taipei, Taiwan

${ }^{\text {al }}$ Also at Department of Physics, The University of Michigan, Ann Arbor MI, United States of America

${ }^{*}$ Deceased 